\title{
Strategies for Efficient Genome Editing Using CRISPR-Cas9
}

\author{
Behnom Farboud,* Aaron F. Severson, ${ }^{t, \pm, s}$ and Barbara J. Meyer*,1 \\ *Howard Hughes Medical Institute and Department of Molecular and Cell Biology, University of California, Berkeley, California \\ 94720-3204, ${ }^{\dagger}$ Center for Gene Regulation in Health and Disease and ${ }^{\ddagger}$ Department of Biological, Geological, and Environmental \\ Sciences, Cleveland State University, Ohio 44115, and §Department of Cellular and Molecular Medicine, Lerner Research Institute, \\ Cleveland Clinic, Ohio 44195 \\ ORCID IDs: 0000-0001-7439-3095 (B.F.); 0000-0002-0300-0669 (A.F.S.); 0000-0002-6530-4588 (B.J.M.)
}

\begin{abstract}
The targetable DNA endonuclease CRISPR-Cas9 has transformed analysis of biological processes by enabling robust genome editing in model and nonmodel organisms. Although rules directing Cas9 to its target DNA via a guide RNA are straightforward, wide variation occurs in editing efficiency and repair outcomes for both imprecise error-prone repair and precise templated repair. We found that imprecise and precise DNA repair from double-strand breaks (DSBs) is asymmetric, favoring repair in one direction. Using this knowledge, we designed RNA guides and repair templates that increased the frequency of imprecise insertions and deletions and greatly enhanced precise insertion of point mutations in Caenorhabditis elegans. We also devised strategies to insert long $(10 \mathrm{~kb})$ exogenous sequences and incorporate multiple nucleotide substitutions at a considerable distance from DSBs. We expanded the repertoire of co-conversion markers appropriate for diverse nematode species. These selectable markers enable rapid identification of Cas9-edited animals also likely to carry edits in desired targets. Lastly, we explored the timing, location, frequency, sex dependence, and categories of DSB repair events by developing loci with allele-specific Cas9 targets that can be contributed during mating from either male or hermaphrodite germ cells. We found a striking difference in editing efficiency between maternally and paternally contributed genomes. Furthermore, imprecise repair and precise repair from exogenous repair templates occur with high frequency before and after fertilization. Our strategies enhance Cas9-targeting efficiency, lend insight into the timing and mechanisms of DSB repair, and establish guidelines for achieving predictable precise and imprecise repair outcomes with high frequency.
\end{abstract}

KEYWORDS Genome editing; DNA repair; homology-directed repair; CRISPR-Cas9; Caenorhabditis elegans

DOGRAMMABLE DNA endonucleases have transformed the analysis of biological processes by enabling targeted genome editing in diverse species (reviewed in Urnov et al. 2010; Joung and Sander 2013; Carroll 2014; Chandrasegaran and Carroll 2016; Knott and Doudna 2018). These nucleases catalyze DNA breaks at specified sequences and trigger precise and imprecise repair outcomes via different pathways (Jasin and Haber 2016). Imprecise repair pathways introduce small-to-large insertions and de-

Copyright (c) 2019 by the Genetics Society of America doi: https://doi.org/10.1534/genetics.118.301775

Manuscript received November 6, 2018; accepted for publication November 29, 2018; published Early Online November 30, 2018.

Supplemental material available at Figshare: https://doi.org/10.25386/genetics. 7390451.

${ }^{1}$ Corresponding author: Howard Hughes Medical Institute and Department of Molecular and Cell Biology, University of California, Berkeley, \#3204, 16 Barker Hall, Berkeley, CA 94720-3204. E-mail: bjmeyer@berkeley.edu letions. Precise repair pathways insert specific DNA changes from either homologous chromosomes or exogenous, homologous templates. Our goal is to improve the frequency and fidelity of genome editing by investigating the rules governing repair pathway choices and repair outcomes.

The programmable endonuclease most widely deployed is the Streptococcus pyogenes CRISPR-associated protein 9 (SpCas9 or Cas9) (reviewed in Mali et al. 2013a; Jiang and Doudna 2017). Cas9 is directed to its DNA target by a guide RNA that pairs with 20 bases of target DNA, called the spacer sequence (Mojica et al. 2009; Garneau et al. 2010; Jinek et al. 2012). The chief constraint limiting target choice is the requirement for an NGG motif to border the DNA sequence that is complementary to the spacer. The NGG motif is called the protospacer adjacent motif (PAM). The Cas9-guide RNA ribonucleoprotein (RNP) 
complex scans the genome, binds to PAM sequences, and melts the adjacent duplex DNA (Sternberg et al. 2014). If the neighboring nucleotides are complementary to the guide RNA, Cas9 undergoes a conformational change that activates its nuclease domains to make a DNA doublestrand break (DSB) (Anders et al. 2014; Sternberg et al. 2015).

These basic principles governing Cas9 targeting led to its widespread usage, but the repertoire of strategies that can be used to achieve desired genomic changes has some limitations. In this study, we develop strategies that overcome several impediments to achieve efficient Cas9 targeting and predictable DSB repair outcomes in the nematode Caenorhabditis elegans. These approaches can be exploited to improve genome editing across diverse plant and animal species.

We demonstrate that both imprecise and precise DNA repair from a single DSB is asymmetric, favoring repair in only one direction. We have exploited this property to establish guidelines for effective PAM choice and design of single-stranded repair templates to achieve high frequency insertion of desired changes within close proximity (30 bp) to a DSB and on a particular side of the DSB. We also devised efficient strategies to insert long non-homologous fragments of DNA ( $\sim 10 \mathrm{~kb})$ at DSB sites and to engineer small specific changes at considerable distance from a DSB (1.5 kb) or to incorporate a series of nucleotide substitutions throughout an entire locus, all without coinserting a selectable marker. These strategies are also useful for inserting DNA in sites such as AT-rich regions that are devoid of potential PAMs. These successes required that we optimize Cas9 delivery methods and guide RNA design.

We also expanded the repertoire of Cas9-dependent coconversion markers to be used in conjunction with the tools to edit targets of choice. These selectable markers, appropriate for diverse nematode species, enable the rapid identification of Cas9-edited animals that are also likely to have desired edits in the targets of choice. This approach is particularly useful when searching for edited targets that fail to cause visible phenotypes.

Finally, we devised and exploited an editing strategy to explore the timing, location, frequency, sex dependence, and categories of DSB repair events. We developed loci with allele-specific targets for Cas9 cleavage that can be contributed from either male or hermaphrodite germ cells during mating. We found that male sperm DNA was generally more permissive to Cas9 editing than hermaphrodite germ cell DNA. The frequency of recovering mutations in the locus of interest is higher if editable alleles of both the target locus and the co-conversion marker are contributed from the same parent during mating. We found that imprecise repair and homology-directed repair (HDR) from homologous chromosomes or exogenous repair templates occurs at an unexpectedly high frequency after fertilization in embryos.

\section{Materials and Methods}

\section{Strains}

Nematode strains were cultured as described previously (Brenner 1974). N2 Bristol was used as the wild-type C. elegans strain and AF16 was used as the wild-type $C$. briggsae strain.

\section{Genome editing in self-fertile hermaphrodites vs. mated hermaphrodites}

Genome editing in C. elegans typically involves the delivery of Cas9 and guide RNA components to the gonad syncytium of young adult self-fertile hermaphrodites that have completed sperm production but have ongoing oocyte production. Loci within hundreds of individual meiotic nuclei in each gonad syncytium have the potential to undergo Cas9dependent cleavage and repair events. This approach has been used for several nematode genome editing studies using Cas9 (Cho et al. 2013; Dickinson et al. 2013; Friedland et al. 2013; Katic and Grosshans 2013; Lo et al. 2013; Tzur et al. 2013; Waaijers et al. 2013; Arribere et al. 2014; Kim et al. 2014; Paix et al. 2014; Zhao et al. 2014; Witte et al. 2015; Farboud and Meyer 2015). Our editing experiments in Figure 1, Figure 2, Figure 3, Figure 4, Figure 5, Figure 6, Figure 7, Figure 8, Figure 9, and Supplemental Material, Figures S1-S4 involved self-fertile hermaphrodites.

For experiments in Figure 10, Figure 11, Figure 12, and Figure S5 that explored the timing, location, frequency, sex dependence, and categories of DSB repair events, we assayed editing in the cross-progeny of mated hermaphrodites. We developed loci with allele-specific targets for Cas9 cleavage that could be contributed from either male or hermaphrodite germ cells during mating. Only the maternal or paternal allele of a locus could be edited (as described later in Materials and Methods). We delivered Cas9 RNPs with or without exogenous repair templates to the gonad syncytium of hermaphrodites that had been mated by males for $24 \mathrm{hr}$. We scored loci of cross-progeny hermaphrodites for imprecise repair and HDR from homologous chromosomes or exogenous repair templates.

\section{Guide RNAs}

For genome editing using single guide RNAs (sgRNAs) produced from DNA expression vectors (Figure 1, Figure 4B, and Figure 7), the relevant target-specific DNA sequences listed in Table S1 were cloned into pRB1017 as described previously (Arribere et al. 2014). For editing using Cas9 RNPs, both the target-specific CRISPR RNA (crRNA) guides and the transactivating crRNA (tracrRNA) were obtained from Dharmacon. All crRNAs, except those used in Figure 7, possessed two 2 '-O-methyl phosphorothioate linkage modifications $(2 \times \mathrm{MS})$ that improves nuclease resistance (Hendel et al. 2015; Dowdy 2017). The different editing efficiencies for the sex-1 AG crRNA used in Figure $7 v$ s. the sex-1 AG crRNA used in Figure 10, Figure 11, and Figure 12 are likely due to the crRNA modification. The efficiencies of all four different 
crRNAs used in Figure 7 are directly comparable, as are the efficiencies of all crRNAs used in the other figures.

\section{Repair templates}

All single-stranded DNA (ssDNA) oligonucleotide repair templates (Table S2) were ordered from Integrated DNA Technologies (IDT) at the $4 \mathrm{nmol}$ Ultramer scale. Single-stranded templates used for examining SNP insertion efficiency $5^{\prime}$ and $3^{\prime}$ of the PAM (Figure 2, A and B, and Figure 3, A and B) were PAGE purified to maximize the fraction of full-length templates.

Long double-stranded DNA (dsDNA) repair templates (Figure 4A, Figure 5, Figure 6, and Figure S2) were purchased as gBlocks from IDT or were made using Gibson assembly reactions to fuse small gBlocks and PCR fragments (Gibson 2011). The templates included silent mutations to eliminate the PAM or to mutate key nucleotides in sequences targeted by guide RNAs. The mutations were designed to prevent Cas9 from cleaving the repair template and the edited genomic locus but not to alter the primary amino acid sequence of the protein encoded by the edited locus. Mating experiments described later in this section confirmed that a single mismatch at the $3^{\prime}$ end of the protospacer can block Cas9 cleavage of the endogenous locus. Sequences for these long dsDNA repair templates are available upon request.

The double-stranded repair template pGEM7z-lir-2 was constructed to examine the relationship between distance from the Cas9 cleavage site and efficiency of HDR from an exogenous template (Figure 4B). A variant of the $3 \mathrm{~kb}$ region centered on the crispr_bf8 (Table S1) target site was cloned into pBluescript $\mathrm{KS}(+)$ using the Gibson assembly of overlapping gBlocks (Gibson 2011). The template carried an altered PAM (GGG to GGA) to prevent cleavage of the template and the edited endogenous locus. On each side of the altered PAM, 10 HindIII sites were created at $\sim 100 \mathrm{bp}$ intervals by introducing mutations that changed 1-4 bp. Approximately $500 \mathrm{bp}$ of uninterrupted lir-2 homologous sequence flanked the core $2 \mathrm{~kb}$ region with the new HindIII sites.

\section{Genome editing using DNA vectors to express Cas9 and guide RNAs}

For editing involving DNA plasmids to express Cas9 and sgRNA guides, injection mixes included $25 \mathrm{ng} / \mu \mathrm{l}$ pRB1017-derived sgRNA plasmid (for each sgRNA), $50 \mathrm{ng} / \mu \mathrm{l}$ pDD162 Cas9 plasmid, and $500 \mathrm{nM}$ dpy-10(gf) or rol-6(gf) single-stranded oligonucleotide repair template. The double-stranded pGEM7z-lir-2 repair template was used at $300 \mathrm{ng} / \mu \mathrm{l}$ (Figure 4B). All plasmids were purified using QIAGEN's (Valencia, CA) Midi Plasmid Purification Kit. Injected P0 worms were allowed to recover for $2-3 \mathrm{hr}$ at $20^{\circ}$ before being transferred to $25^{\circ}$. After 3 days, Rol and/or Dpy worms were picked to individual plates, allowed to produce self-progeny, and screened for mutations at loci of interest.

\section{Genome editing using Cas9 RNP complexes}

Cas9 RNPs were prepared for injection as described previously (Paix et al. 2015). Briefly, $20 \mu \mathrm{l}$ injection mixes consisting of $8.75 \mu \mathrm{M}$ Na-HEPES, pH 7.5, $115 \mu \mathrm{M}$ KCl, $15 \mu \mathrm{M}$ Cas9-NLS (QB3, University of California, Berkeley Core facility), $15 \mu \mathrm{M}$ dpy-10 crRNA guide (crispr_bf32), $15 \mu \mathrm{M}$ for each crRNA guide to a locus of interest, $42 \mu \mathrm{M}$ tracrRNA, and $500 \mathrm{nM}$ dpy-10 single-stranded oligonucleotide repair template were assembled on ice. Any additional ssDNA or dsDNA repair templates were used at a final concentration of $500 \mathrm{nM}$ or $350 \mathrm{ng} / \mu \mathrm{l}$, respectively. RNP cocktails were made and delivered by pipetting all reagents into a tube on ice, incubating at $37^{\circ}$ for $15 \mathrm{~min}$, centrifuging at $13,000 \times g$ for $1 \mathrm{~min}$, loading into a microinjection needle, and injecting into gonads of young adult hermaphrodites. Injected P0 hermaphrodites were treated as described above.

\section{Co-conversion strategy using ben-1 in C. elegans and C. briggsae}

We developed ben-1 as a co-conversion marker for both C. elegans and C. briggsae (Figure 8, A and B). ben-1 encodes $\beta$-tubulin, and ben-1 mutations confer resistance to benzimidazole (benomyl), which binds to $\beta$-tubulin, inhibits microtubule polymerization, and induces paralysis, uncoordinated (Unc) movement, and a dumpy (Dpy) morphology in both strains (Chalfie and Thomson 1982; Driscoll et al. 1989).

To establish the benzimidazole concentrations required to induce the desired phenotypes in $C$. elegans and $C$. briggsae, we placed young adult N2 or AF16 worms on nematode growth (NG) agar plates containing 5, 7.5, 10, 12.5, or $15 \mu \mathrm{M}$ benzimidazole; incubated them at $25^{\circ}$ for 3 days; and examined their $\mathrm{F}_{1}$ progeny. Growth on $7.5 \mu \mathrm{M}$ benzimidazole caused the expected phenotypes in C. elegans, while growth on $12 \mu \mathrm{M}$ benzimidazole was required in C. briggsae.

The Unc and Dpy phenotypes resulting from benzimidazole treatment were efficiently suppressed by loss-offunction ben-1 mutations induced by injecting Cas9 RNP mixtures that included the crispr_bf41 guide and BF-2035 repair template in $C$. elegans and the crispr_bf39 guide and BF-2036 repair template in C. briggsae (Tables S1 and S2). Both guide RNAs had an AA at the 3 ' end of their targetspecific sequences to yield efficient but not fully optimal editing. Use of less efficient ben-1 guides translated into higher co-conversion frequencies for the genes of interest. Both single-stranded repair templates introduced a premature in-frame stop codon at the homologous locus to knock out gene function. To simplify detection of precise edits, repair templates were designed to introduce a new $\mathrm{XbaI}$ site into the $C$. elegans ben-1 locus and a new NdeI site into the C. briggsae locus.

Worms injected with repair templates and Cas9 RNPs targeting ben-1 and a locus of interest were allowed to recover for $2-3 \mathrm{hr}$ at $20^{\circ}$ and then transferred to $25^{\circ}$ for 2-3 days. Mobile non-Unc non-Dpy animals (produced from 
successful ben-1 editing) were picked to individual plates, allowed to produce self-progeny at $25^{\circ}$, and screened for edits at loci of interest. Growth at $25^{\circ}$ allows detection of both heterozygous and homozygous ben-1 mutations, which are semidominant or dominant at $25^{\circ}$ but recessive at $15^{\circ}$.

In our experience with multiple targets and co-conversion markers, Cas9-mediated genome editing appears less efficient in $C$. briggsae than in $C$. elegans. Therefore, we recommend injecting a larger number of worms when editing the $C$. briggsae genome.

\section{Co-conversion selection using zen-4 in C. elegans}

We developed zen-4(+) as a co-conversion marker to enable the selection of fully wild-type progeny produced by zen-4 (cle10ts) lethal mutants injected with Cas9 RNPs and repair templates (Figure 9, A and B). The zen-4(cle10ts) mutation causes embryonic lethality within $30 \mathrm{~min}$ of upshift from the $15^{\circ}$ permissive temperature to the $25^{\circ}$ nonpermissive temperature. The selectable zen $4(+)$ repair event restores both DNA and protein sequences to wild-type.

For co-conversion using zen-4(+) repair templates, young zen-4(cle10ts) adult hermaphrodites grown at $15^{\circ}$ were injected quickly at $20^{\circ}$ with a mixture containing Cas9 RNPs with zen-4 guide RNAs (crispr bf46; Table S1) and the wild-type repair template (BF-2046; Table S2) to convert the three zen-4(cle10ts) SNPs to fully wild-type sequences (Figure 9). After injection, worms were allowed to recover on NG agar plates at $15^{\circ}$ for $24 \mathrm{hr}$. After $24 \mathrm{hr}$, the injected P0 worms were transferred to new NG agar plates and both sets of plates (those with the injected P0s and those with $\mathrm{F}_{1}$ embryos laid for $24 \mathrm{hr}$ ) were transferred to $25^{\circ}$ for 3 days. Both zen-4(+)/zen-4(cle10ts) and zen-4(+)/zen$4(+)$ embryos hatched and developed into phenotypically normal, fertile adults. In contrast, zen-4(cle10ts) homozygous mutant animals that hatched prior to the $25^{\circ}$ shift developed to adulthood but were Unc, scrawny, and sterile, making them clearly distinguishable from wild-type worms. Mutant embryos that failed to hatch prior to the $25^{\circ}$ temperature shift were dead after the shift. Confirmation for the conversion of one or both of the zen-4(cle10ts) alleles to zen-4(+) was achieved by digesting a PCR-amplified zen-4 amplicon with AluI (Figure 9A). The zen-4(cle10ts) amplicons were cleaved, but the zen-4(+) amplicons remained intact. Loci of interest were examined for edits in the zen-4(+) worms.

\section{Screening strategies to assess editing at loci of interest}

In all experiments presented, co-conversion protocols were used to enrich for worms with genome editing events at loci of interest (Arribere et al. 2014; Kim et al. 2014; Ward 2015). P0 animals injected with reagents to edit a co-conversion marker and a locus of interest were allowed to lay progeny for 2-3 days, and $F_{1}$ progeny expressing the phenotype of a co-conversion marker-rol-6(gf), dpy-10(gf), ben-1(lf), or zen- $4(+)$-were screened for edits at loci of interest, either after laying progeny or without laying progeny, according to the experimental design.

When using $d p y$-10 as a co-conversion marker, we found, as expected, that precise repair of one allele with an exogenous repair template that introduces the nucleotide changes found in dpy-10(cn64) recapitulates the dominant left-hand roller (Rol) phenotype of $d p y-10$ (cn64)/+ animals. However, imprecise repair of a single allele of $d p y-10$ or heteroallelic combinations of different $d p y-10$ lesions can yield a variety of Rol, Dpy, or Dpy-Rol phenotypes (Levy et al. 1993). We examined animals exhibiting that spectrum of $d p y-10$ phenotypes for mutations in our gene of interest.

Imprecise repair events identified by Sanger sequencing: After individual $\mathrm{F}_{1}$ rol-6(gf) animals laid $\mathrm{F}_{2}$ progeny on agar plates for 2-3 days, they were transferred to separate wells of a 96-well plate and then lysed using protocols described previously (Figure 1) (Farboud and Meyer 2015). Locus-specific PCR and Sanger sequencing of PCR products were performed on DNA from lysed $\mathrm{F}_{1} \mathrm{~s}$ using the oligonucleotides in Table S3. Imprecise repair was detected by either the presence of overlapping traces beginning near the DSB site (heterozygous imprecise edits) or single mutant traces (homozygous imprecise edits). $\mathrm{F}_{2}$ progeny from $\mathrm{F}_{1}$ worms carrying heterozygous imprecise repair events were cloned, and their loci of interest were amplified by PCR and then assayed by Sanger sequencing to identify $\mathrm{F}_{2}$ homozygous mutants and determine the exact sequence changes caused by imprecise repair.

Templated HDR and imprecise repair events identified by Sanger sequencing: To assay genome editing events, dpy-10 mutant $\mathrm{F}_{1}$ progeny were lysed in individual wells of a 96-well plate (Figure 2, Figure 3, Figure 4A, Figure 7, Figure 10, Figure 11, Figure 12, and Figure S5). Loci of interest were PCR amplified using the primers in Table S3 and analyzed by Sanger sequencing. Sequencing chromatograms revealed whether loci had heterozygous programmed HDR events (double traces at the site of SNP insertion), homozygous HDR events (single mutant trace), or no editing events (single wild-type trace). For all eight figures, homozygous and heterozygous imprecise repair was detected as in Figure 1, except clonal analysis of $\mathrm{F}_{2}$ worms was not used to identify indel endpoints.

Templated HDR events identified by PCR and restriction fragment length polymorphism analysis: To determine the length of repair tracts templated from dsDNA repair templates harboring HindIII sites at $100 \mathrm{bp}$ intervals (Figure 4B), $1.6 \mathrm{~kb}$ fragments were amplified on the $3^{\prime}$ and the $5^{\prime}$ side of the PAM using the primers listed in Table S3. Amplicons were digested with HindIII and the restriction fragments were resolved through electrophoresis on a $2.5 \%$ agarose gel. The banding pattern revealed the distance of the terminal-most SNP from the Cas9 DSB. If the entire repair template had been inserted, the amplicon would be reduced to $\sim 10$ 100 bp fragments and the $\sim 600$ bp fragment from the 
homology arm that lacks HindIII sites. If partial insertion occurred, the size of the largest fragment would reveal the length of DNA lacking HindIII sites. Subtraction of the largest fragment length from the full $1.6 \mathrm{~kb}$ amplicon length revealed the approximate distance from the DSB that the HDR insertion stopped.

Templated HDR events identified by insert-specific PCR followed by Sanger sequencing: To monitor the insertion of large DNA fragments (Figure 5, Figure 6, and Figure S2), individual $\mathrm{F}_{1}$ dpy-10 mutant worms that laid $\mathrm{F}_{2}$ progeny on individual agar plates were transferred to separate wells of a 96-well plate and lysed. For Figure 5, A-C, and Figure S2, PCR was performed using primers that yielded product only when the desired genomic insert was present. One primer annealed to the inserted sequence, while the other primer annealed to adjacent chromosomal sequences absent from the repair template. This approach prevented false-positive amplification of repair templates present in extrachromosomal DNA arrays. A second PCR reaction using primers that bound just outside the two Cas9 target sites yielded product only from chromosomes lacking large insertions.

The $F_{2}$ progeny of $F_{1}$ animals that tested positive for the insertion were also cloned, allowed to lay $\mathrm{F}_{3}$ progeny, and then genotyped to identify animals that were homozygous for the desired insert. Homozygotes produced insert-specific PCR amplicons, but no PCR amplicons from primers specific for DNA lacking the insert. The accuracy of insertion was confirmed by Sanger sequencing across the entire inserted genomic region of homozygotes from at least four independent insertion events. Insertions were precise, with no sequence changes near the DSBs.

For Figure 5D, PCR was performed on DNA from $\mathrm{F}_{1} \mathrm{~s}$ to amplify across the genomic insertion site, with one primer annealing to genomic sequences absent from the repair template. Sanger sequencing was then performed to assess the presence of desired small deletions and point mutations. $\mathrm{F}_{2}$ progeny of $F_{1} s$ that were heterozygous for the desired mutations were cloned and retested by Sanger sequencing to identify homozygous mutants. The full set of desired sequence changes was present in each independent insertion event ( 8 insertions for the deletions and 18 for the point mutations).

For Figure 6, two sets of PCR reactions were performed on lysed $\mathrm{F}_{1} \mathrm{~s}$ from each strain to test for insertions at DSB A and DSB B. For each reaction, one primer annealed to genomic sequence located outside of the bounds of the repair template and a second primer annealed to novel DNA within the repair template. Precise repair at A and B was indicated by properly sized PCR A and PCR B amplicons. Precise repair at A, but not $B$, was shown by a properly sized PCR A amplicon and lack of or incorrect size of a PCR B amplicon. Precise repair at B but not A was indicated by a properly sized PCR B amplicon and lack of or incorrect size of a PCR A amplicon. Analysis by Sanger sequencing of homozygous strains with each class of repair from the three PAM orientations confirmed that the properly sized PCR amplicons reflected precise repair at a DSB junction.

\section{Examining the effects of mating on genome editing efficiency}

Matings were set up between males and hermaphrodites with genotypes shown in Figure 10, Figure 11, Figure 12, and Figure S5 by placing 2 L4 hermaphrodites and 10 males on NG agar plates with small ( $\sim 0.5 \mathrm{~cm}$ diameter) spots of OP50 bacteria. Worms were allowed to mate at $20^{\circ}$ for $24 \mathrm{hr}$ prior to microinjection. Young adult hermaphrodites were then transferred to new NG agar plates without bacteria prior to mounting for microinjection. Swollen spermathecae visible at the time of injection indicated that hermaphrodites had mated successfully. After microinjection and recovery at $20^{\circ}$ for $3 \mathrm{hr}$, P0 worms were transferred to individual NG agar/ OP50 plates and incubated for 3 days at $25^{\circ}$. Presence of $F_{1}$ male offspring provided further evidence that mating had occurred. Both Rol and/or Dpy hermaphrodites and males produced by successfully mated mothers were transferred to individual wells of a 96-well plate for lysis and genotyping, as described above.

In our experience using $d p y-10$ as a co-conversion marker, half or more of injected self-fertile P0 hermaphrodites yielded a high frequency of $\mathrm{F}_{1}$ progeny with Dpy or Rol phenotypes. However, for experiments in Figure 11 and Figure 12 in which hermaphrodites were mated with males prior to injection and the $d p y$ - 10 allele from only one parent could be targeted by Cas9, only a quarter or less of the injected $\mathrm{P} 0$ animals produced $\mathrm{F}_{1}$ progeny with Dpy or Rol phenotypes.

The genomic feature that permitted selective targeting of maternal vs. paternal chromosomes was a single base-pair change in a critical region of the spacer DNA required for pairing with the guide RNA. When targeting $X$-specific loci using a guide that could only pair with the male spermspecific allele, $\mathrm{F}_{1}$ males produced by the cross were examined by DNA sequence analysis to determine whether the inherited maternal $X$ chromosome had been edited via the mismatched guide. We found that male progeny lacked edited $X$ chromosomes, indicating that the single-base mismatch in the spacer seed sequence of the maternal genome prevented its cleavage.

\section{Strategy for scoring inter-homolog HDR events}

For Figure 11 and Figure 12, classifying DNA changes as the result of inter-homolog HDR events required distinguishing cross-progeny from self-progeny. By experimental design, animals that arose from inter-homolog HDR events would be Rol or Dpy hermaphrodites that carried homozygous sex-1 GG alleles. However, in some crosses, Rol or Dpy sex-1 GG homozygous animals could have been self-progeny of sex-1 GG homozygous hermaphrodites that failed to mate with sex-1 AG males. Thus, we only considered Rol or Dpy sex-1 GG homozygous animals to be the result of inter-homolog HDR events if they were cross-progeny. 
For Figure 11, A and D, and Figure 12A, the RNA guides permitted only the $d p y-10$ AG allele contributed from the male sperm to be edited. Therefore, all Rol or Dpy animals had to be cross-progeny, and Rol or Dpy sex-1 GG homozygous animals should have arisen from cleavage of the sex-1 AG allele and repair using the sex-1 GG allele contributed by the other parent.

In Figure $11 \mathrm{~B}$ and Figure 12B, the editable $d p y-10$ AG allele was contributed by the mother. Thus, all Rol or Dpy hermaphrodites that were homozygous for the paternal sex-1 GG allele could be classified as cross-progeny that underwent inter-homolog HDR events.

In Figure 11C, the frequency of inter-homolog HDR events was estimated by assuming that all assayed $F_{1}$ Dpy or Rol progeny were cross-progeny. We considered this assumption to be valid based on our results from experiments in Figure $11, \mathrm{~A}, \mathrm{~B}$, and D, in which cross-progeny could be identified unambiguously. In those experiments, all assayed Dpy or Rol progeny were cross-progeny, suggesting that all matings in the experiments of Figure 11 were fully successful. Several guidelines helped ensure that matings were successful and that the assayed Dpy or Rol progeny were cross-progeny. First, when mounting PO worms for microinjection, the mated hermaphrodites were examined using differential interference contrast microscopy to confirm successful matings by the presence of engorged spermathecae. Second, prior to assaying Rol or Dpy $\mathrm{F}_{1}$ animals, the $\mathrm{F}_{1}$ offspring were examined to confirm that nearly half of the $\mathrm{F}_{1}$ offspring were males, an indication of fully successful mating prior to microinjection.

For Figure 11, A and B, and Figure 12, A and B, repair events classified as inter-homolog HDR required that the cleaved target in the haploid genome of one gamete be repaired using the homologous chromosome in the haploid genome of the other gamete, thereby generating an embryo that was homozygous for a SNP in sex-1. Primers that anneal $\sim 200$ bases from the sex-1 SNP were used for PCR and Sanger sequencing to confirm the genotype of the offspring. It was formally possible that a cleaved chromosome could have been repaired imprecisely to yield a deletion that would have prevented the PCR primers from annealing to the deleted sequences in the targeted sex-1 locus. In that case, only sequences from the uncut chromosome would be amplified, and the sex-1 locus would be incorrectly classified as homozygous for the SNP due to interhomolog HDR. To rule out such false-positive results, $10 \mathrm{~F}_{1}$ progeny that appeared to have undergone inter-homolog HDR at sex- 1 were allowed to produce $\mathrm{F}_{2}$ progeny. A total of $16 \mathrm{~F}_{2}$ progeny from each of the 10 different $F_{1}$ animals were lysed and their DNA sequenced to confirm that the edits were indeed homozygous. We found that PCR never failed to amplify the loci and all the DNA sequences were consistent with inter-homolog HDR events. In these sex-1 experiments, deletions in the vicinity of the SNP do not cause any lethality and we found no death among $\mathrm{F}_{2}$ progeny. Thus, our analysis of $\mathrm{F}_{2}$ progeny would not have missed false-positive inter-homolog HDR events due to lethality.

\section{Data availability}

Strains are available upon request. Supplemental material, including five figures and three tables, available at Figshare: https://doi.org/10.25386/genetics.7390451.

\section{Results and Discussion}

\section{Imprecise repair at Cas9 cleavage sites is asymmetric, favoring changes $5^{\prime}$ of the PAM}

Analysis of imprecise repair outcomes at Cas9 DSBs in sex-1 and lir-2 revealed a polarity to the insertion and deletion of sequences that occurred in the absence of exogenous repair templates. Changes were preferentially introduced $5^{\prime}$ of the PAM (Figure 1). Even when imprecise repair caused deletions $3^{\prime}$ of the PAM, the deletions were substantially shorter in the $3^{\prime}$ direction than in the $5^{\prime}$ direction on the same DNA strand. The propensity of repair to favor changes $5^{\prime}$ of the PAM that is true for nematodes has been found in mammals as well (Mali et al. 2013b; van Overbeek et al. 2016; Richardson et al. 2018), providing the opportunity to use $C$. elegans as a model to understand general rules underlying such directionality and apply them to achieve changes in desired locations.

\section{Precise repair from homologous single-stranded oligonucleotide templates is directional and its efficiency is dictated by the choice of repair template strand}

To determine whether HDR is also asymmetric, we compared repair outcomes using single-stranded oligonucleotide templates that matched either the protospacer strand or the spacer strand of three different loci: lir-2, sex-1, and an intergenic site on chromosome I (Chr. 1 site) (Figure 2, A and B). For both classes of repair templates, two adjacent SNPs were positioned at a site corresponding to the chromosomal DSB site. These two adjacent SNPs constitute polymorphism b. Additional SNPs were also positioned $10 \mathrm{nt}$ from the DSB in both $5^{\prime}$ and $3^{\prime}$ directions (Table S2). As a convention, we defined the positions of edits at endogenous loci as $5^{\prime}$ or $3^{\prime}$ of the PAM.

For both protospacer and spacer strand repair templates, repair events including polymorphism b at DSB sites were frequent at all three endogenous loci (15-47\% of dpy-10 mutants), but a striking preference was found for insertion of SNPs $5^{\prime}$ of the PAM when the protospacer strand was used for the repair template. As shown in Figure 2A, $12-37 \%$ of the $d p y-10$ mutants had a SNP inserted 5' of the PAM from the protospacer template but $0 \%$ of worms had a SNP inserted 3' of the PAM. However, if the spacer strand was used for the repair template, insertion of a SNP 3' of the PAM was strongly favored. We found that $6-13 \%$ of $d p y-10$ mutants had a $3^{\prime}$ polymorphism inserted from the spacer strand template, but 
lir-2(+)

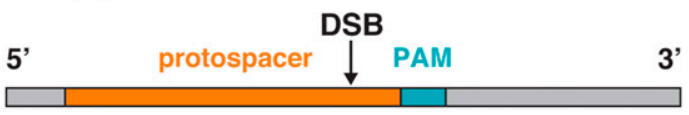

lir-2 mutant alleles

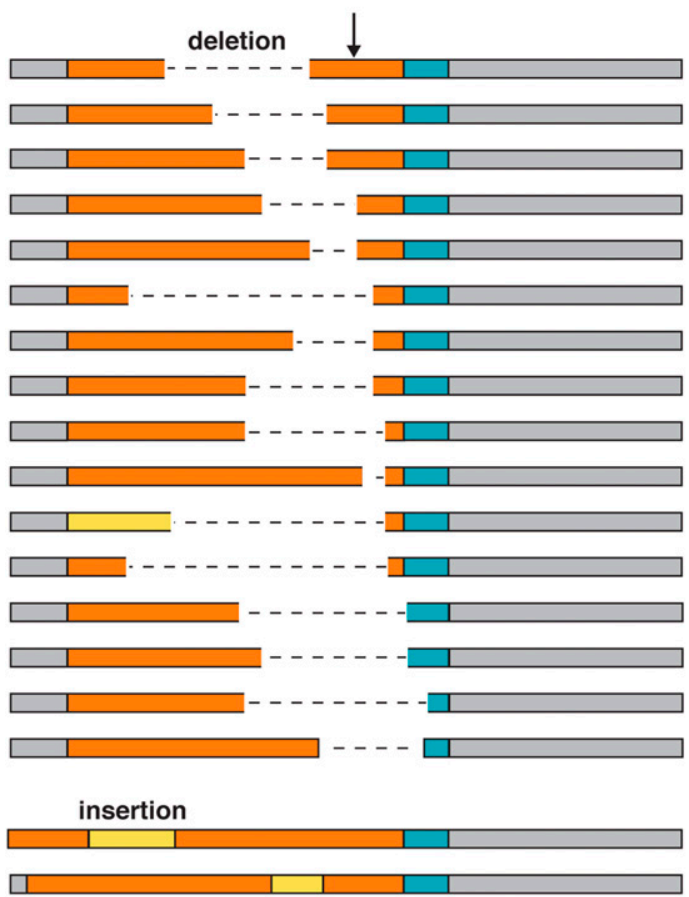

$\operatorname{sex}-1(+)$

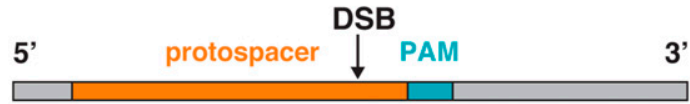

sex-1 mutant alleles

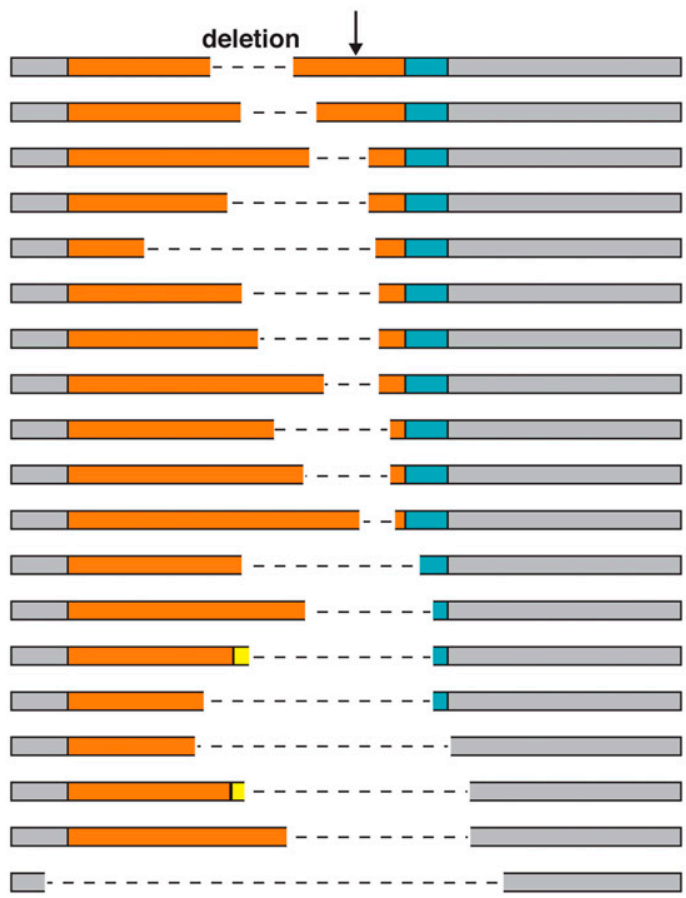

Figure 1 Imprecise repair at Cas9 DSBs causes asymmetric insertion and deletion of DNA sequences in regions 5' of the PAM. Diagrams depict the protospacer strand from wild-type lir-2 (left) and sex-1 (right) genes, featuring the locations of PAMs (blue), DSBs ( $\downarrow$ ), and target-specific sequences (orange). Below are protospacer strand diagrams showing deletions (dashed lines) and insertions (yellow) caused by imprecise repair at the two genes. All diagrams are drawn to scale. These examples are representative of the lir-2 and sex-1 changes found in edited animals in which rol-6(gf) was the co-conversion marker. For the last two lir-2 repair examples, only insertions occurred. Because the DNA is drawn to scale and the same amount of DNA is shown for all examples, the gray region to the left of the protospacer was shortened to accommodate the length of the insertion. Imprecise repair was biased toward regions located 5' of the PAM.

only 0-4\% of $d p y-10$ mutants had a 5 ' polymorphism inserted (Figure 2B). Because we detected both imprecise repair and HDR at high frequency at the predicted Cas9 cleavage sites, we are certain that the lack of HDR in either the $5^{\prime}$ or $3^{\prime}$ direction was not caused by the failure of Cas9 to cleave DNA (Figure 2, A and B).

The dramatic polarity in repair can be explained by a repair process that proceeds via a synthesis-dependent strand annealing mechanism (SDSA), as diagrammed in the models of Figure 2C (Sekelsky 2017). The 3' end of the cleaved spacer strand in the endogenous locus can anneal readily with the complementary protospacer strand repair template and prime DNA synthesis from the template, thereby incorporating polymorphisms only at the DSB and $5^{\prime}$ of the PAM (Figure 2C, left). Similarly, the $3^{\prime}$ end of the cleaved protospacer strand in the endogenous locus can anneal with the complementary spacer strand repair template and prime DNA synthesis from the template, thereby incorporating polymorphisms only at the DSB and $3^{\prime}$ of the PAM (Figure $2 \mathrm{C}$, right). This SDSA model is rigorously supported by the repair outcomes from our two complementary sets of experiments. An SDSA repair model has also been proposed by others in the context of repair from a single-stranded break generated by a Cas 9 mutant variant having only one of its two single-stranded nickases (Davis and Maizels 2014, 2016) and in the context of repair from Cas9 DSBs (Kan et al. 2017; Paix et al. 2017) and meganucleases (Kan et al. 2014).

The occasional insertion of SNPs 5' of the PAM when a spacer strand repair template was used can be explained by the 3' exonucleolytic activity for the Cas9 nickase RuvC (see model in Figure S1) (Jinek et al. 2012; Jiang et al. 2015; Zuo and Liu 2016; Stephenson et al. 2018). This nickase resects ssDNA in vitro up to 10 nucleotides in the $3^{\prime}-5^{\prime}$ direction on the strand it nicks (Stephenson et al. 2018). Since polymorphism a is only $10 \mathrm{bp}$ from the DSB, the RuvC nickase should occasionally resect the endogenous DNA just past the location of the polymorphism and permit primer extension from the spacer repair template to incorporate this polymorphism into the endogenous locus at low frequency, as we observed. 
A

Repair outcomes with protospacer repair template genomic DNA

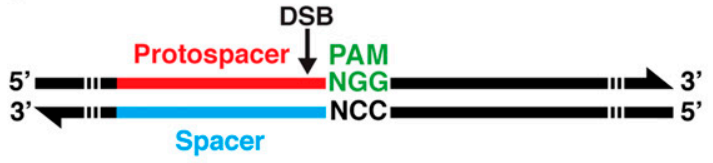

Protospacer strand repair template $100 \mathrm{nt}$

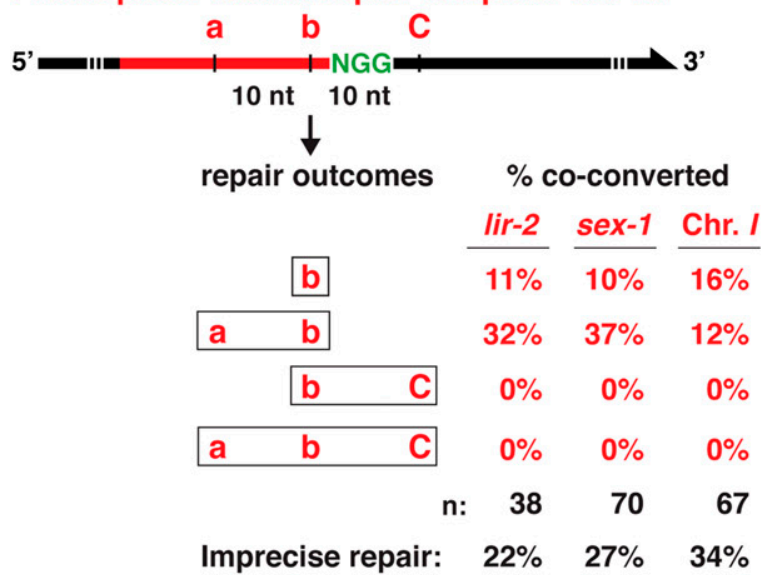

C Models:

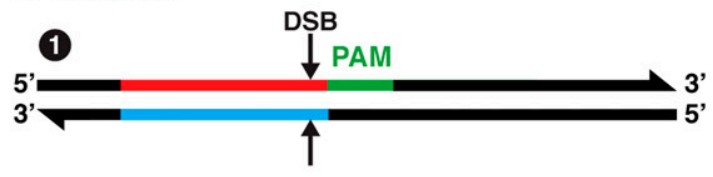

Cas9 DSB
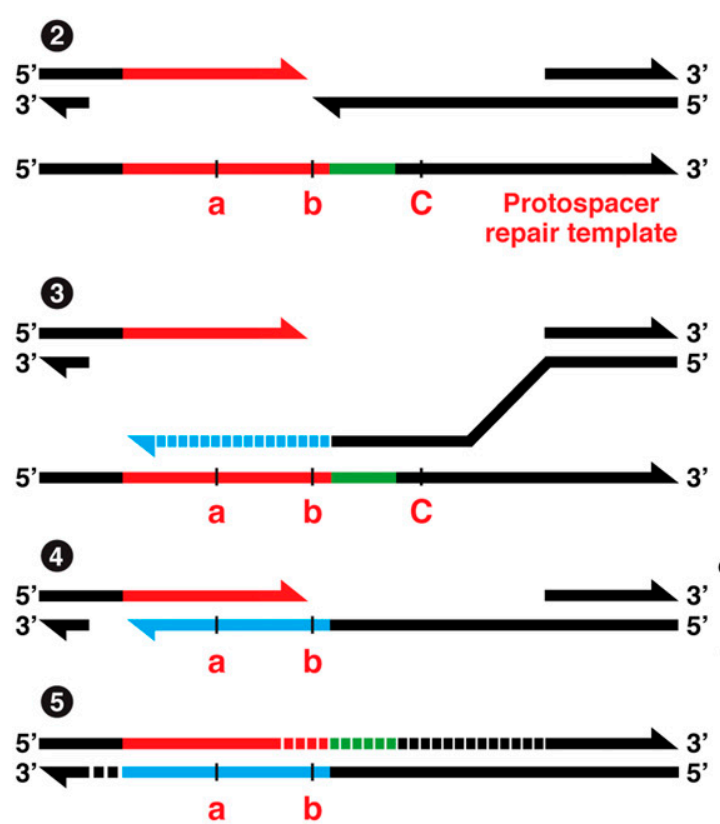
annealing to template, primer extension 5' to 3' resection template
dissociation,
annealing
, of extended
end to other
DSB side completion of repair synthesis, ligation

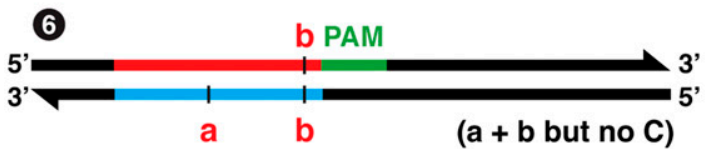

B

Repair outcomes with spacer repair template genomic DNA

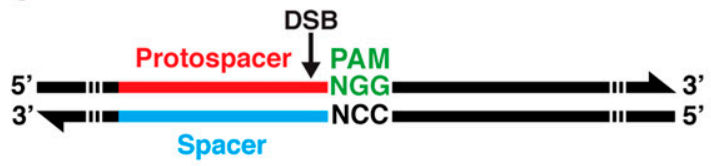

Spacer strand repair template $100 \mathrm{nt}$

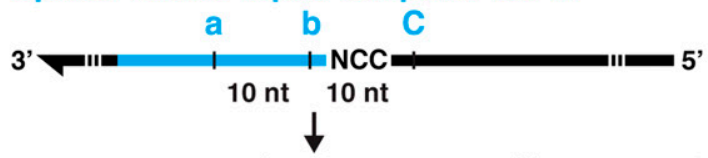

repair outcomes $\quad \%$ co-converted
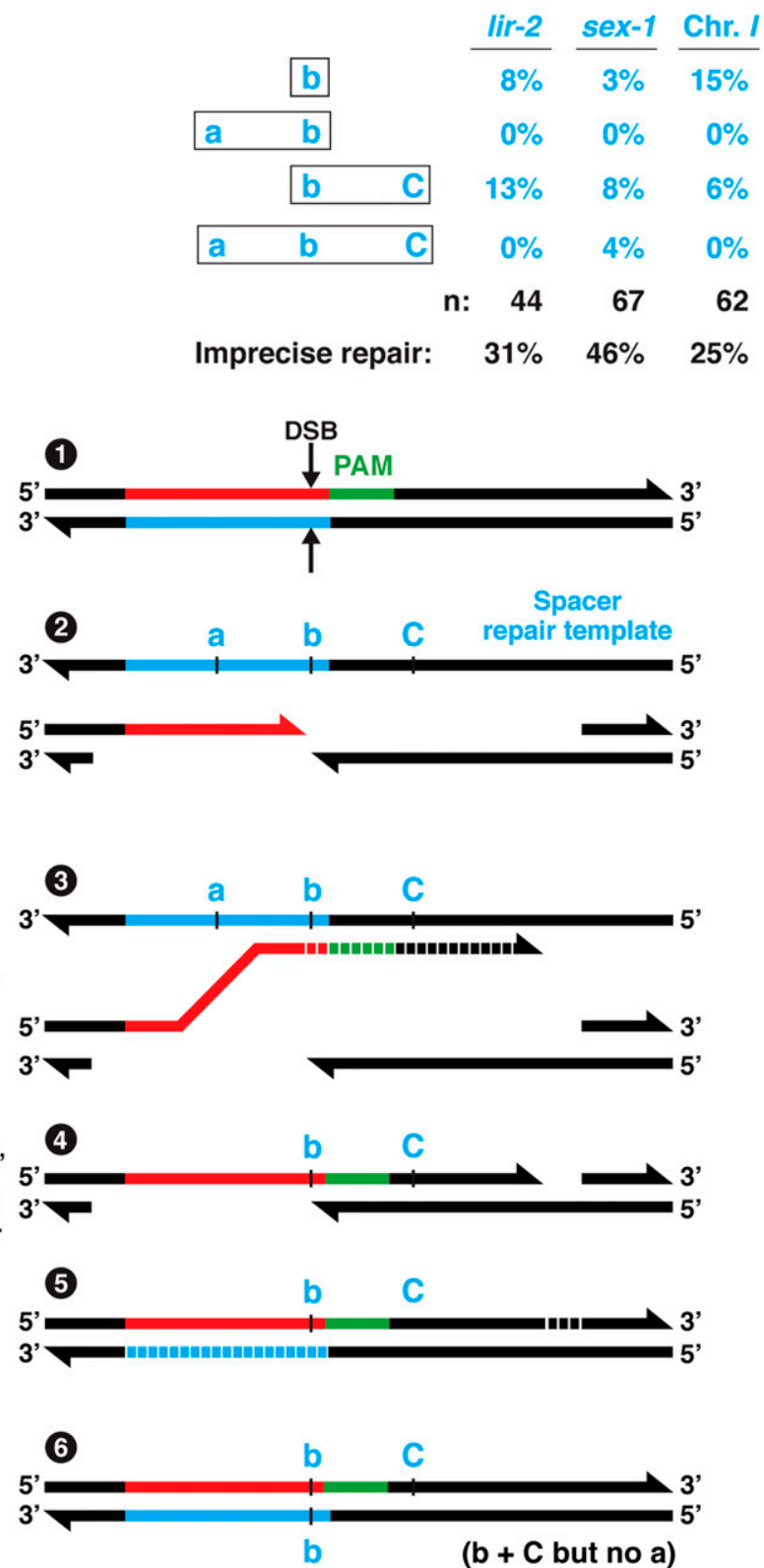

Figure 2 Polarity of HDR from single-stranded templates is dictated by the choice of repair template strand. (A and B) Analysis of genome editing experiments at three loci (lir-2, sex-1, intergenic chromosome I site) comparing HDR outcomes using Cas9 RNPs and single-stranded repair templates of either $(A)$ the protospacer strand or (B) spacer strand. Polymorphisms were located in the repair templates at the predicted endogenous DSB sites (SNP b) and at sites both 5' (SNP a) and 3' (SNP C) of the PAM (Table S2). Percentages represent the frequencies of diagrammed repair outcomes at each locus 
We addressed two additional issues about DSB repair from single-stranded repair templates: (1) whether the presence of polymorphisms on both sides of the DSB, as in Figure 2, blocks the template from being used efficiently for repair; and (2) whether all polymorphisms within 30 bp of a DSB will be inserted with similar frequency relative to those closest to the DSB. If repair is very local, the repair process may not insert the more distant changes in the repair template.

We addressed both topics by using single-stranded repair templates designed to insert polymorphisms immediately adjacent to the chromosomal DSB site (polymorphism d) and also in one direction from the DSB, in $10 \mathrm{nt}$ intervals (Figure 3). Similar to the pattern observed using repair templates with symmetric changes, repair templates corresponding to the protospacer strand resulted in efficient insertion of polymorphisms directly over the DSB (30 and 26\%) and $5^{\prime}$ of the PAM (14\%), but not $3^{\prime}$ of the PAM (0\%) (Figure 3A). Remarkably, incorporation of all four changes in the $5^{\prime} \mathrm{di}$ rection was more efficient (12\%) than incorporation of only a subset of the changes $(2 \%)$ ( $P<0.02$, chi square). In contrast, repair templates corresponding to the spacer strand (Figure 3B) resulted in efficient insertion of polymorphisms at the DSB (39 and 16\%) and $3^{\prime}$ of the PAM (22\%), but not $5^{\prime}$ $(0 \%)$. Insertion of all four changes in the $3^{\prime}$ direction $(11 \%)$ was on par with insertion of only a subset of changes (11\%), in contrast to the protospacer template result.

Several generalizations emerged from this set of experiments. Insertion of polymorphisms specifically at the DSB site is highly efficient regardless of whether the single-stranded repair template corresponds to the protospacer strand or spacer strand. However, insertion of SNPs $5^{\prime}$ of the PAM is far more efficient if the repair template corresponds to the protospacer strand, while insertion of SNPs $3^{\prime}$ of the PAM is much more efficient if the repair template corresponds to the spacer strand. Therefore, the choice of repair template strand is critical for ensuring the insertion of polymorphisms in the desired locations. Finally, when SNPs were inserted either 5' or $3^{\prime}$ of the PAM, a tendency prevailed to incorporate all of the SNPs in one direction, not just a subset of the SNPs, particularly for the protospacer repair template.

The precise repair outcomes we observed were independent of whether the strand of the repair template was noncoding (sex-1, lir-2), coding (zen-4), or intergenic (site on chromosome I) (Table S2, Materials and Methods). Others have suggested that successful insertion of polymorphisms was higher if the coding strand of the gene was used as the repair template rather than the noncoding strand (Katic et al. 2015). However, our reexamination of the published repair outcomes revealed that the polarity can be explained by the location of the polymorphism relative to the PAM and is therefore consistent with the strong trend in our experiments.

\section{For HDR at a single Cas9 DSB, a double-stranded repair template was less efficient than a single- stranded template}

Optimization of genome editing strategies requires a comparative analysis of HDR efficiency with single-stranded $v s$. double-stranded repair templates. We initiated this comparison by repeating the experiment of Figure 2A with a linear double-stranded repair template that was identical in sequence to the 100 nt sex-1 single-stranded HDR template. To our surprise, we observed no insertion of SNPs at the sex-1 locus in the 144 animals that were edited at the $d p y-10$ locus (Figure 4A). Instead, $75 \%$ of the $d p y-10$ mutants had undergone imprecise repair at the sex-1 locus, indicating that Cas9 cleavage was effective but that HDR from the double-stranded template was completely ineffective. Furthermore, the lack of HDR was not caused by the double-stranded repair template being destroyed by Cas9 cleavage because the template carried SNPs in the sequence required by the guide RNA (Materials and Methods and subsequent experiments).

In a second experiment, we used a $1 \mathrm{~kb}$ linear doublestranded repair template to sex-1 that included the same SNPs as the $100 \mathrm{bp}$ template but replaced the $40 \mathrm{bp}$ homology arms with 490 bp arms (Figure 4A). Results with this template were more successful but still not robust. The frequency of imprecise repair was reduced to $39 \%$, and the frequency of HDR elevated: $4 \%$ of $d p y-10$ mutants had SNP insertions at the DSB site and 1-2\% also had SNP insertions on either side of the DSB, but not both sides. Thus, repair with linear double-stranded repair templates was considerably less efficient than repair with single-stranded templates.

A third experiment assessed the maximum distance from the DSB that SNPs were inserted into lir-2 from a doublestranded repair template (Figure 4B). The $4 \mathrm{~kb}$ HDR template was embedded in a circular plasmid, had 500 bp homology arms, a SNP that disrupted the PAM (hence Cas9 binding), and 10 polymorphisms positioned at $\sim 100$ bp intervals on both sides of the DSB. Each polymorphism had 1-4 bp substitutions that created a HindIII site for monitoring HDR. HDR outcomes were infrequent ( $6 \%$ of 380 dpy-10 mutants) and exhibited a striking polarity: insertions occurred up to 900 bp from the DSB but primarily in only one direction, $5^{\prime}$ of the PAM. The exception was a polymorphism that was inserted $100 \mathrm{bp}$ from the DSB in the opposite direction. Thus, the strategy of using a single Cas9 cleavage site and a

relative to the total number of mutants ( $\mathrm{n}$ ) expressing the co-conversion marker in that experiment. Both protospacer and spacer repair templates promoted insertion of SNPs at the DSB site, but the protospacer strand template promoted insertion of SNPs exclusively $5^{\prime}$ of the PAM, while the spacer strand template promoted insertion of SNPs preferentially $3^{\prime}$ of the PAM. Frequency of imprecise repair is also provided for each experiment. For these experiments, $d p y$-10 (gf) was the co-conversion marker. When precise templated HDR occurred at the $d p y$ - 10 locus, the animals exhibited a dominant Rol phenotype and a recessive Dpy phenotype. If imprecise repair occurred at $d p y-10$, animals exhibited only a recessive Dpy phenotype. All animals with any of these phenotypes were examined for SNPs. (C) Models show proposed steps in the HDR process for protospacer (left) and spacer (right) strand repair templates based on a repair mechanism involving SDSA. Numbers refer to sequential steps. 
A

Protospacer strand HDR template: repair 5' of PAM

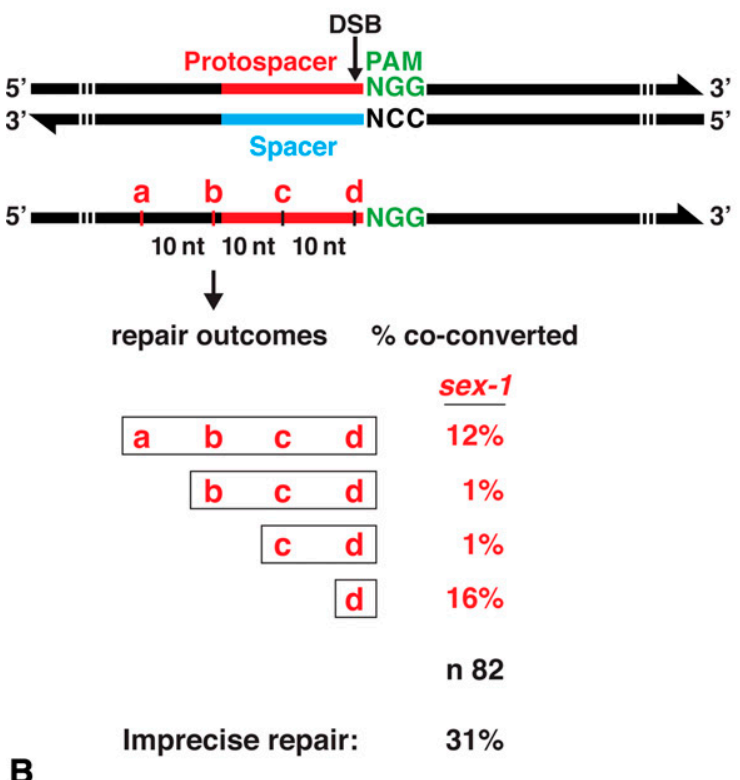

Protospacer strand HDR template: repair 3' of PAM

Spacer strand HDR template: repair 5' of PAM
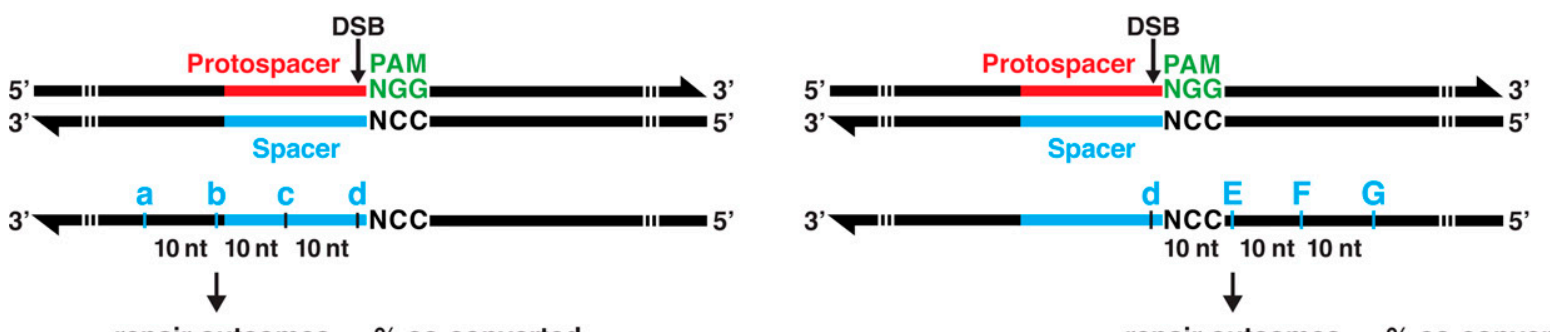

repair outcomes $\%$ co-converted

repair outcomes \% co-converted

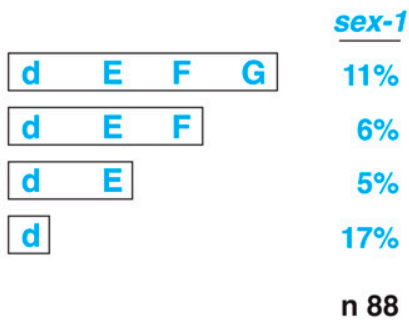

Imprecise repair: $\quad 39 \%$

Imprecise repair: $\quad 23 \%$

Figure 3 Frequency of SNP insertion from single-stranded repair templates as a function of distance from the DSB. (A and B) Shown is the frequency of diagrammed HDR outcomes at the sex-1 locus using Cas9 RNPs and either (A) single-stranded protospacer strand or (B) spacer strand repair templates with two SNPs located at the DSB (polymorphism d) and single SNPs in one direction from the DSB, in 10 nt intervals. Insertion of polymorphisms at the DSB was highly efficient regardless of the repair template strand, but protospacer strand templates promoted insertion of SNPs only $5^{\prime}$ of the PAM, while spacer strand templates promoted insertion of SNPs only $3^{\prime}$ of the PAM. Repair outcomes revealed a strong tendency to incorporate all SNPs in one direction from the PAM, not just a subset of SNPs. Repair templates include a 40 nt homology arm, an adjacent 30 nt region with SNPs, a PAM, and a $50 \mathrm{nt}$ homology arm. The percentages of repair outcomes reflect the number of the diagrammed outcomes relative to the total number of $d p y-10$ mutants $(n)$ in the experiment. Percentage of imprecise repair is also indicated.

double-stranded repair template to edit the genome yielded only a low frequency of HDR events. These events were too directional and limited to permit insertion of large DNA fragments with multiple polymorphisms.

A final set of experiments assessed whether a large non-homologous DNA fragment (9300 bp) could be inserted into the genome at a single DSB site. We evaluated doublestranded repair templates with three different sets of 500 bp homology arms. We found that $0 \%$ of 576 edited animals had an insert of the 9300 bp DNA fragment, thus reinforcing the conclusion that large dsDNA fragments are generally difficult to insert into the genome using only one 


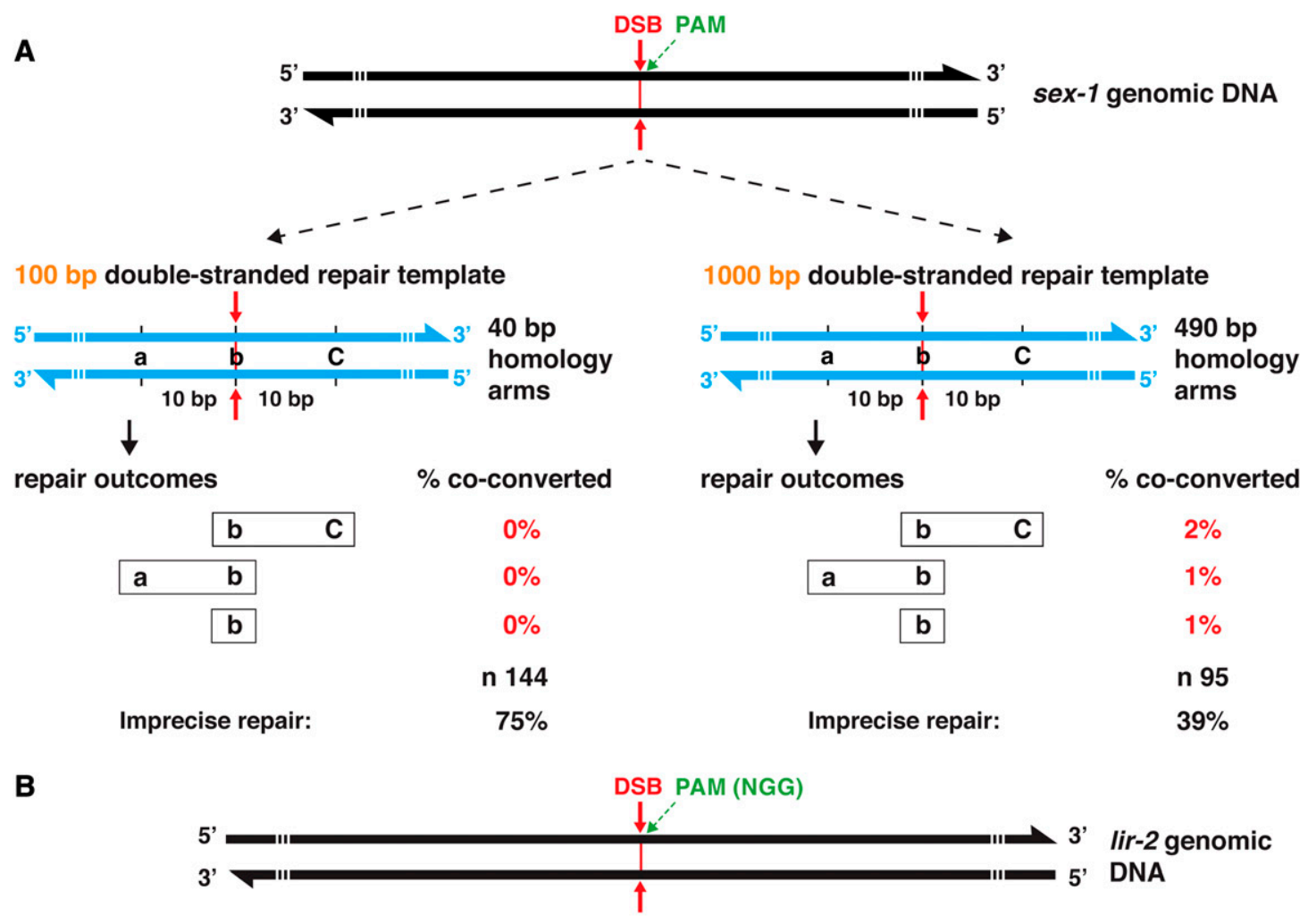

3000 bp double-stranded repair template

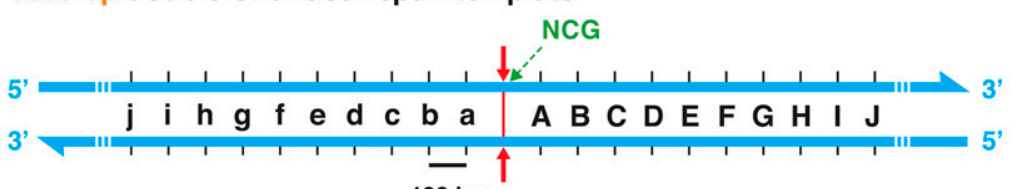

1

repair outcomes

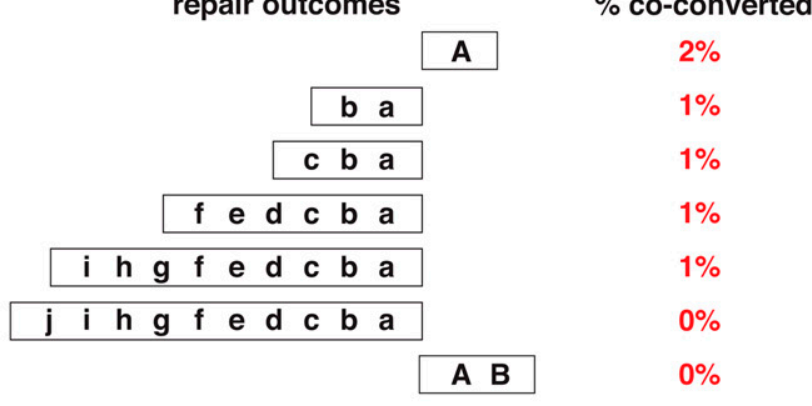

No incorporation of SNPs B to $\mathrm{J}$

n 380

Figure 4 HDR from double-stranded repair templates was asymmetric relative to the DSB and less efficient than repair from single-stranded templates. (A, left) The experiment in Figure 2A was reproduced in design except for the use of a linear $100 \mathrm{bp}$ double-stranded repair template instead of a $100 \mathrm{nt}$ single-stranded repair template. Unexpectedly, no HDR was observed, although $75 \%$ of the $d p y-10$ mutants had undergone imprecise repair at the DSB in sex-1. (A, right) The experiment in the left panel was repeated, except the linear double-stranded repair template (1 kb) had 490 bp homology arms instead of $40 \mathrm{bp}$ arms. The outcome was more successful, but the frequency of HDR was greatly reduced compared to the frequency of HDR in Figure 2A with single-stranded repair templates. (B) Analysis of SNP insertions relative to the distance from the DSB. The 4 kb double-stranded repair template had 500 bp homology arms and 10 polymorphisms at $\sim 100$ bp intervals on both sides of the DSB. Each of the 20 polymorphisms created a HindllI restriction site for analyzing repair outcomes. Precise HDR was infrequent and exhibited a striking directionality in which infrequent insertions occurred up to 900 bp from the DSB, but predominantly only $5^{\prime}$ of the PAM. For A and B, the number of animals with the diagrammed insertion was expressed as the percentage of the total number of $d p y-10$ Dpy or Rol mutants (n). Cas9 RNPs were used in A, and DNA expression vectors were used for Cas9 and the guide RNAs in B. The $4 \mathrm{~kb}$ repair template was introduced as plasmid DNA. 

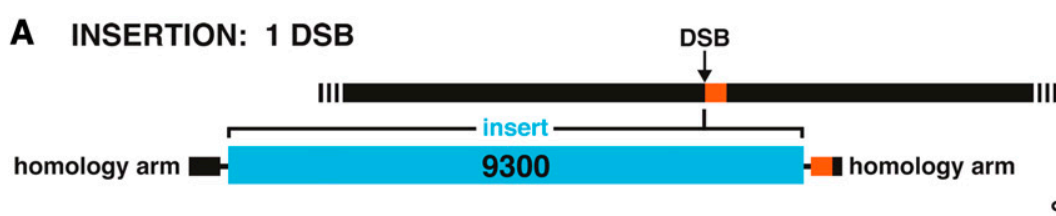

$\%$ co-converted (n)
$0 \%(192)$

B DELETION AND INSERTION: 2 DSBS DSB DSB

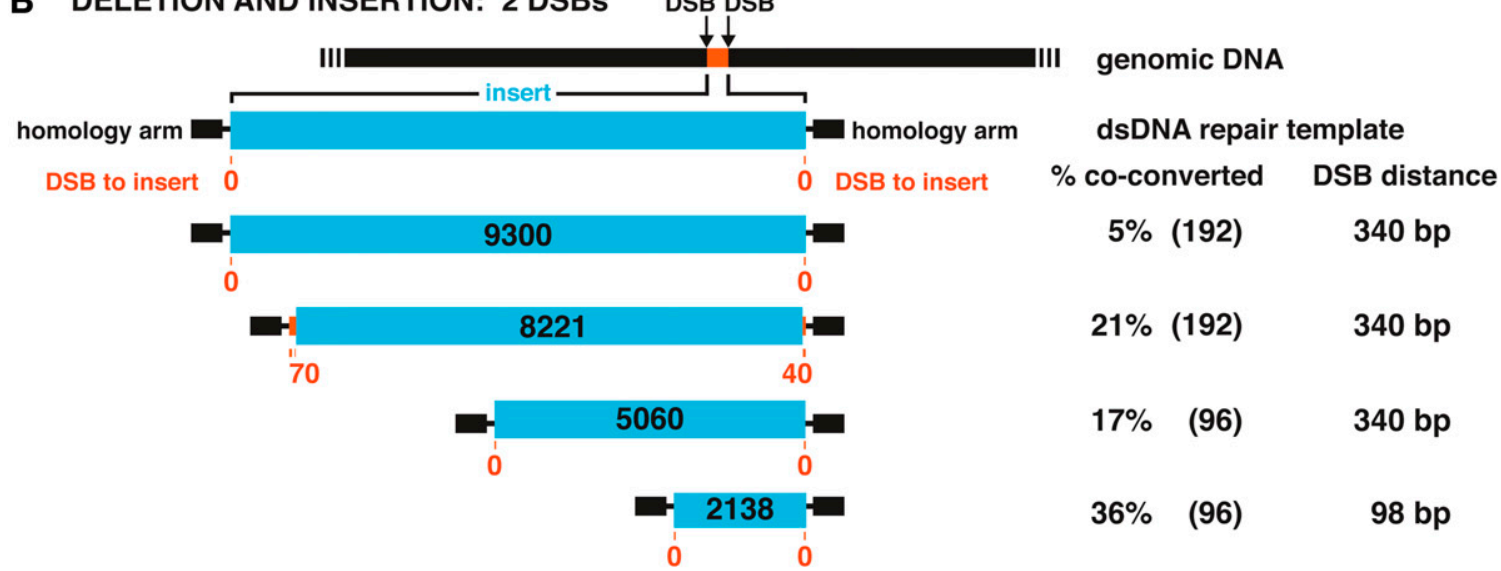

genomic DNA

dsDNA repair template

dsDNA repair template

$5 \%(192) \quad 340 \mathrm{bp}$

$21 \%(192) \quad 340 \mathrm{bp}$

$17 \% \quad(96) \quad 340 \mathrm{bp}$

$36 \% \quad(96) \quad 98 \mathrm{bp}$

genomic DNA

dsDNA repair template

\% co-converted DSB distance

15\% (192) 2826 bp

$12 \%(192) \quad 2826$ bp

$9 \%(192) \quad 2826$ bp

$18 \%(192) \quad 573 \mathrm{bp}$

D INSERTION: 2 DSBS

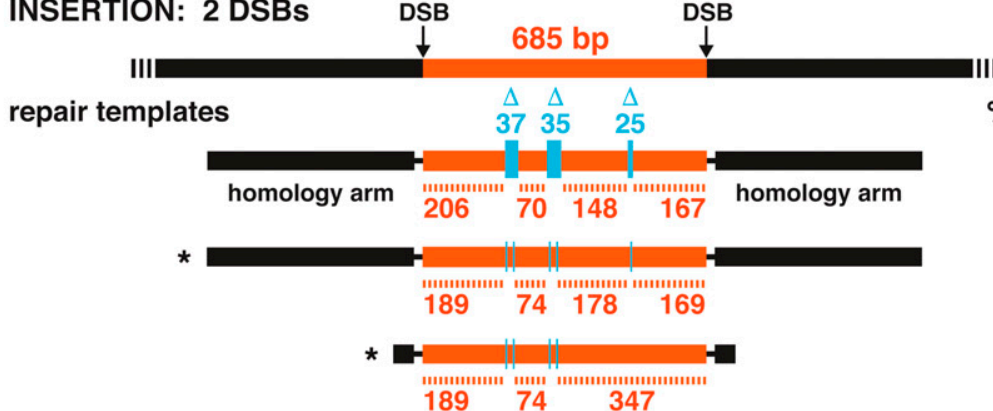

genomic DNA

$\%$ co-converted

$8 \% \quad(95)$

DSB distance

$13 \%(136)$

* Of co-converted animals, all had the full set of changes from the repair template.

Figure 5 Two neighboring DSBs promote insertion of large DNA fragments. (A) Insertion of a 9300 bp reporter transgene was not successful in experiments involving a single Cas9 DSB and a double-stranded repair template with 500 bp homology arms (black and orange/black) flanking the DSB. See also results in Figure S2. (B) Insertion of long DNA fragments (blue) was efficient in dpy-10 co-conversion experiments when two neighboring DSBs were made using two different guide RNAs. In this experimental design, the DNA between the DSBs (340 or 98 bp) was deleted when the large DNA fragment was inserted. Double-stranded repair templates with 500 bp homology arms (black) were injected as plasmid DNA along with the Cas9 RNPs. For insertion of the 8221 bp reporter, the repair template included the standard 500 bp homology arms and additional 70 or 40 bp regions of homology to the DNA between the DSBs, making the total length of deleted DNA 230 not $340 \mathrm{bp}$. For all experiments in this figure, the term "DSB to insert" refers to the distance between the DSB and the novel DNA to be inserted. PAM orientation was IN/IN for the top three examples and IN/OUT for the bottom 
DSB (Figure 5A and Figure S2). However, a recent article reported some success using one DSB to insert $1 \mathrm{~kb}$ fragments by using a double-stranded repair template with singlestranded overhangs (Dokshin et al. 2018).

\section{Efficient strategies to insert large DNA fragments at wide-ranging distances from DSBS}

To overcome the strong polarity in HDR events repaired from double-stranded templates, the inefficiency in inserting polymorphisms that are distant from a DSB, and the inefficiency in inserting large, non-homologous fragments into the genome, we explored whether two nearby DSBs made using two different guide RNAs simultaneously would facilitate insertion of long DNA fragments (Figure 5A). Two DSBs have been used to make chromosomal rearrangements, including inversions, translocations, and large deletions (Blasco et al. 2014; Chen et al. 2014; Choi and Meyerson 2014; Ghezraoui et al. 2014; Maddalo et al. 2014; Paix et al. 2014; Iwata et al. 2016; Dejima et al. 2018). We found that adding a second DSB at a distance of $340 \mathrm{bp}$ from the initial DSB (Figure 5A and Figure S2) enabled us to insert a 9300 bp fragment from a doublestranded template with reasonable efficiency (5\%) (Figure 5B).

This double DSB strategy also worked well using a variety of linear and circular double-stranded repair templates with 500 bp homology arms to insert fluorescent reporters: $21 \%$ dpy-10 mutants had precise insertions of $8221 \mathrm{bp}, 17 \%$ had precise insertions of $5060 \mathrm{bp}$, and $36 \%$ had precise insertions of $2138 \mathrm{bp}$ (Figure 5B). In all cases, the DNA between DSBs (340 or 98 bp) had been deleted and the desired DNA inserted. Our approach using two nearby DSBs to incorporate long DNAs is sufficiently robust and precise that selection strategies are not needed to enrich the number of candidates to screen for full-length insertions.

A slight variation on our strategy enabled the insertion of desired DNA at long distances from DSBs without deleting any intervening DNA. The variation is highly useful when the target sites for editing lack nearby sequences needed for effective Cas9 guides or when a series of different substitutions are desired simultaneously along the length of DNA (Figure 5, C and D). The adaptation depends solely on the design of the repair template. For example, we inserted a 1275 bp fragment with good efficiency in various locations within the 2826 bp region of DNA between two DSBs in xol-1 simply by using different $4101 \mathrm{~kb}$ HDR templates that included the entire 2826 bp homologous region with the 1275 bp non-homologous insert placed at various locations within the homologous region (Figure 5C). The non-homologous DNA could be inserted as far as 1968 bp from one of the DSBs. The purpose was to insert 24 copies of the binding site for the MS2 phage coat protein into different introns for the goal of performing live imaging of transcription.

By a similar strategy, we deleted three short regions (25$37 \mathrm{bp}$ ) within an intron of xol-1 and made $3 \mathrm{bp}$ substitutions at five different locations within the intron to define the binding sites used by an RNA binding protein in vivo. In the latter experiment, all the desired SNPs were incorporated into each of the edited introns. Thus, the high efficiency of inserting long, full-length stretches of DNA into the genome using two DSBs provides the flexibility to perform genome editing at multiple sites in a large region of genomic DNA.

Others reported that repair template homology arms $>50$ bp can reduce the efficiency of HDR (Paix et al. 2014). We have obtained good HDR efficiency with 500 bp homology arms but also tested 50 bp homology arms to ask whether the efficiency could be improved. We found that it was not (Figure 5D).

\section{For HDR induced by two Cas9 cleavage sites, relative orientation of PAMs influences efficiency of HDR}

Since successful genome editing using a single Cas9 cleavage site required a specific PAM orientation relative to the site of editing and the repair template strand, we explored the significance of PAM orientation on HDR efficiency when two simultaneous DSBs were employed. Cas9 binds PAMs tightly both in vitro and in vivo and remains bound after DNA cleavage. Each DSB generates two DNA ends: the end with the PAM and the end without the PAM. These two unique DNA ends are likely to be processed differently during DSB repair and may support different rates of HDR.

To understand the effect of PAM orientation on HDR using two DSBs, we examined repair rates at the same genomic locus in three strains that differed only by the orientation of PAMs at the two Cas 9 target sequences. The PAMs were arranged in an OUT/OUT, IN/IN, or IN/OUT orientation (Figure 6). The PAM OUT orientation left the PAM on the chromosome end after DNA excision. The PAM IN orientation placed the PAM within the DNA sequence excised from the chromosome.

example (see Figure 6 legend for the orientation key). (C) Insertion of long DNA fragments at wide-ranging distances from both DSBs was accomplished by a variation in the double-stranded repair template. The template included all the DNA between the DSBs (orange) and positioned the new DNA to be inserted (blue) at different locations within the DNA spanning the DSBs. In this design, no DNA was deleted at the endogenous locus; only new DNA sequences were inserted. DSB locations for the last example differed from those in the other three examples. PAM orientation was OUT/OUT for the top three examples and OUT/IN for the bottom example. (D) Using a similar design of double-stranded repair template as in C, genome editing was accomplished at multiple sites in a large region of genomic DNA. In the top example, small deletions (blue) were introduced along the DNA, while in the bottom example, 3 bp substitutions (blue) were inserted at five different locations. PAM orientation was IN/IN for the three examples. For A-D, the number of animals with the diagrammed insertion was expressed as the percentage of total $d p y-10$ Rol or Dpy mutants given in parenthesis. Homology arms for all repair templates were $500 \mathrm{bp}$, except for the bottom example in D, which had $50 \mathrm{bp}$ homology arms. All repair templates for A except the 3128 bp template were injected as purified plasmid DNA along with the Cas9 RNPs. Repair templates in C and D were linear dsDNAs. All diagrams were drawn to scale. 


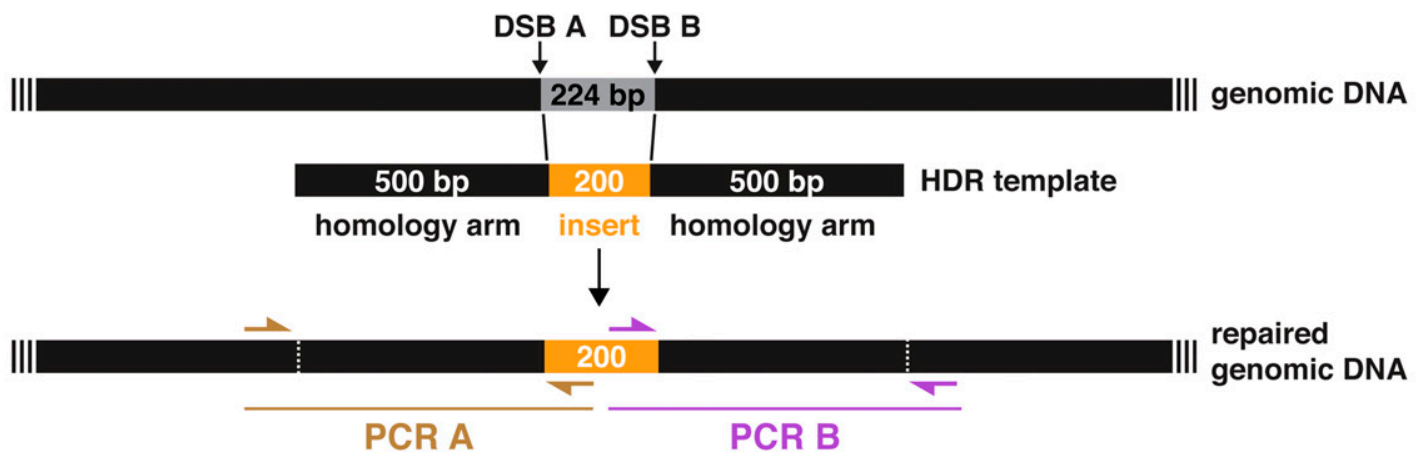

Protospacer PAM

DSB GGCTGACATCCAGTAGACGGAGG

Target A CCGACTGTAGGTCATCTGCCTCC

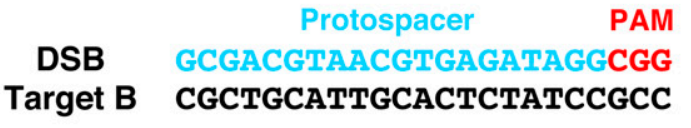

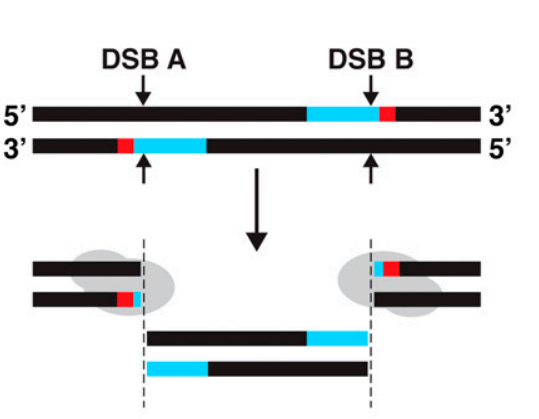

Repair template

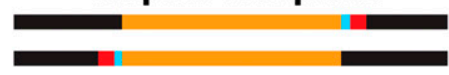

OUT / OUT

Precise repair at

$A$ and $B$

Precise repair at A not B

\section{Precise repair at $B$ not A}

n 152

$6 \%$

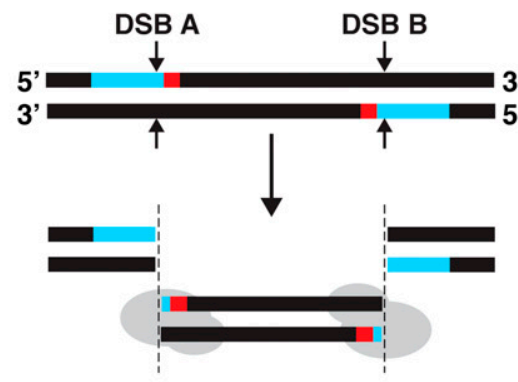

Repair template

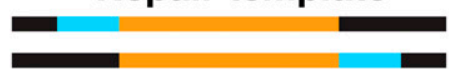

IN / IN

$12 \%$

$4 \%$

$3 \%$

$2 \%$

$11 \%$

224

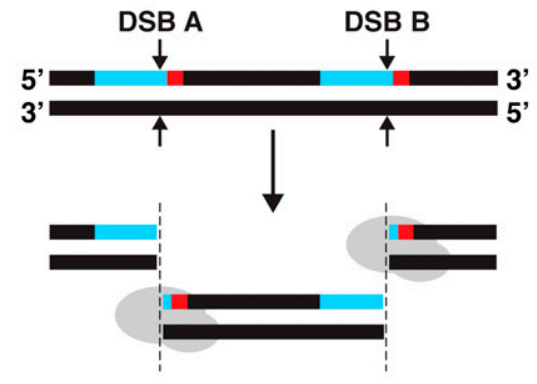

Repair template

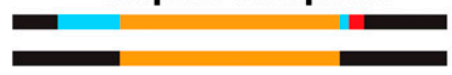

IN / OUT

Figure 6 Orientation of PAMs at two nearby Cas9 cleavage sites influences HDR efficiency. Cas9 was directed simultaneously to two locations in close proximity (224 bp, target A and target B) to make two DSBs and thereby stimulate insertion of an exogenously supplied homologous repair template. The effect of relative PAM orientation on HDR efficiency was assessed by analyzing three strains that had identical sequences at the insertion sites except for the orientation of targets A and B. The PAM IN orientation placed the PAM within the DNA sequence excised from the chromosome. The PAM OUT orientation left the PAM on the chromosome end after DNA excision. The dsDNA repair templates had 500 bp homology arms corresponding to genomic sequences adjacent to DSB A and DSB B. The three repair templates used for the three different strains were identical except for the cleavage remnants of targets $A$ and $B$ included in the template, as shown in the diagram. All three templates included the same novel DNA to be inserted (orange). Precise HDR resulted in replacement of the 224 bp region (gray) between DSB A and DSB B with 200 bp of exogenous DNA sequence from the 
Since the three strains harbor the same two unique Cas9 target sequences, strain-specific differences in repair frequencies should reflect differences in PAM orientations and not differences in Cas9 recognition and cleavage efficiency.

Orienting both PAMs outward (OUT/OUT) resulted in the most efficient rate of HDR: $21 \%$ of $d p y-10$ hermaphrodites had the templated changes (Figure 6). In this OUT/OUT orientation, the PAMs would be retained on the chromosome ends after DNA cleavage. In the other extreme orientation, the IN/IN orientation, $12 \%$ of the dpy-10 mutants had the templated sequences. For this orientation, the PAMs would be on the excised piece of DNA. The least efficient repair occurred with one PAM in the IN orientation and the other in the OUT orientation (IN/OUT), resulting in one PAM on the excised DNA end and the other on the chromosome end. Only $4 \%$ of Rol or Dpy mutants had the precise insert. A similar HDR pattern held true for the much larger repair template used in Figure S4 in which HDR from the IN/IN orientation was $19 \%$ but only $7 \%$ from the IN/OUT orientation.

No permutation of PAM orientations resulted in the total failure of HDR for the fully matched set of experiments in Figure 6, or the more random experiments in Figure 5. Thus, although the most successful strategy for efficient HDR insertions is to use Cas9 targets that position both PAMs in the OUT orientation, other orientations can also work, just not as effectively. The low efficiency of achieving insertions with either the IN/OUT or OUT/IN orientation of PAMs likely accounts in part for the difficulty in inserting large segments of DNA using a single Cas9 guide and hence only one DSB, which naturally causes the cleaved ends to have an IN/OUT orientation.

A relevant set of experiments involving different PAM orientations explored the effect of overhangs made from Cas9 variants, each with one nickase activity, either RuvC or HNH (Bothmer et al. 2017). Consistent with our results, the experiments showed that loci with two overhangs having the PAM OUT/OUT orientation gave a higher frequency of imprecise repair than loci with two overhangs having the PAM IN/IN orientation.

Our experiments recovered not only animals with precisely repaired DNA at both DSBs, but also animals with precisely repaired DNA at one DSB and imprecisely repaired DNA at the other DSB (Figure 6). No pattern emerged that correlated the IN or OUT PAM orientation with precise $v s$. imprecise repair at either of the two DSB junctions.

\section{Optimizing guide RNA design and Cas9 delivery}

Efficient genome editing requires optimization of the guide RNA design to ensure a high frequency of Cas9-induced DSBs.
We showed previously for $C$. elegans that guide RNAs having a GG sequence at the $3^{\prime}$ end of their target-specific sequences were the most effective for achieving targeted mutagenesis (Farboud and Meyer 2015). We revisited the importance of the GG guide sequence because we changed our protocol for delivering Cas9 to germline nuclei from injecting DNA expression vectors encoding Cas9 and guide RNAs to the more effective protocol of injecting pre-assembled Cas9/guide RNPs. Using both delivery protocols, we compared directly the efficiency of genome editing using guides having GG, AG, CG, or UG at their 3' ends (Figure 7). To do so, we first performed genome editing to make four isogenic strains in which sex-1 genomic DNA had been changed by a single nucleotide substitution to convert the penultimate $3^{\prime} \mathrm{G}$ of the protospacer to either $\mathrm{A}, \mathrm{C}$, or $\mathrm{T}$.

With the DNA expression vector delivery approach, only the sex-1 GG RNA guide induced imprecise repair (68\%) at the endogenous sex-1 locus of animals edited at dpy-10. In contrast, RNP injections were more permissive, but the GG RNA guide was still far more effective at genome editing: while $71 \%$ of GG RNA guides induced imprecise repair at the sex-1 locus, only $28 \%$ of AG guides, $18 \%$ of CG guides, and $12 \%$ of UG guides induced sex-1 changes. Thus, while genome editing in C. elegans, as in other organisms, can be accomplished with guides lacking a $\mathrm{G}$ as the penultimate $3^{\prime}$ nucleotide of the target-specific sequence, the efficiency of mutagenesis in C. elegans is much higher if the guides have a penultimate $\mathrm{G}$.

\section{Efficient co-conversion marker to edit genomes of evolutionarily diverged nematodes}

Most co-conversion markers for C. elegans (dpy-10, rol-6, sqt-1) reside on chromosome II, making it inconvenient to edit another gene on chromosome II because the edited gene will have an unwanted linked visible marker (Arribere et al. 2014). Furthermore, mutations in neither rol-6 nor dpy-10 have strong dominant phenotypes in C. briggsae, thus limiting their utility as co-conversion markers in this diverged sister species (15-30 MYA).

To overcome both problems, we developed ben-1 as a co-conversion marker effective for both $C$. elegans and $C$. briggsae (Figure 8, A and B). ben-1, a gene on chromosome III, encodes $\beta$-tubulin. While developing $C$. elegans genome editing protocols, we showed previously that ben-1 loss-offunction mutations made using zinc-finger nucleases (ZFNs) and transcription activator-like effector nucleases (TALENs) were dominant for conferring resistance to the drug benomyl (Wood et al. 2011; Lo et al. 2013). Benomyl binds to $\beta$-tubulin, inhibits microtubule polymerization, and causes

repair template (orange). Only the PAM (red) and protospacer (blue) are shown on the diagrams of target DNA and repair templates. Sanger sequencing of PCR products from the brown primer pair for target $A$ and the purple pair for target $B$ was used to screen co-converted Dpy or Rol animals for precise repair at A and/or B (see Materials and Methods). Percent (\%) co-converted $F_{1} s$ with precise or imprecise repair at Cas 9 targets is shown for each PAM orientation. $\mathrm{n}$ is the total number of co-converted $\mathrm{F}_{1} \mathrm{~s}$ scored by PCR. The percent of repair from the OUT/OUT orientation was significantly greater than that for the IN/IN orientation ( $P=0.046$, chi square) or the IN/OUT orientation $\left(P<10^{-4}\right.$, chi square). The percent of repair from the IN/IN orientation was significantly greater than that for the IN/OUT orientation $(P=0.007$, chi square). 


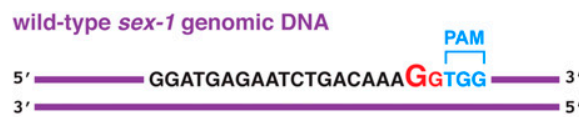

\begin{tabular}{|c|c|c|c|c|}
\hline $\begin{array}{r}\text { targeted } \\
\text { sex-1 alleles }\end{array}$ & --AAAGGTGG-- & --AAAAGTGG-- & --AAACGTGG-- & --AAATGTGG-- \\
\hline Guides & $\mathrm{G}_{\mathrm{G}}$ & $\mathrm{AG}_{\mathrm{G}}$ & $\mathrm{C}_{\mathrm{G}}$ & $\mathbf{U}_{\mathbf{G}}$ \\
\hline & \multicolumn{4}{|c|}{$\%$ co-converted } \\
\hline Cas9 DNA & $\begin{array}{l}68 \% \\
\text { (n 80) }\end{array}$ & $\begin{array}{c}0 \% \\
\text { (n 110) }\end{array}$ & $\begin{array}{r}0 \% \\
\text { (n 62) }\end{array}$ & $\begin{array}{r}0 \% \\
\text { (n 95) }\end{array}$ \\
\hline Cas9 RNP & $\begin{array}{l}71 \% \\
\text { (n 95) }\end{array}$ & $\begin{array}{l}28 \% \\
\text { (n 47) }\end{array}$ & $\begin{array}{l}18 \% \\
\text { (n 49) }\end{array}$ & $\begin{array}{l}12 \% \\
\text { (n 49) }\end{array}$ \\
\hline
\end{tabular}

Figure 7 Optimization of guide RNA design and Cas9 delivery method. Imprecise repair outcomes in isogenic sex-1 strains differing only by a single base-pair change enabled comparison of the effectiveness of four RNA guides differing only in the penultimate nucleotide at the $3^{\prime}$ end of the target-specific sequence. Effectiveness of two different Cas9/guide delivery methods was compared for each guide: RNPs vs. DNA expression vectors. Using the DNA expression vector delivery approach, only the GG guide induced imprecise repair. Cas9 RNPs were more permissive, but the GG guide was still more effective than the other guides. Percentages represent the frequencies of imprecise repair events relative to the total number of $d p y-10$ co-converted $\mathrm{F}_{1} \mathrm{~s}(\mathrm{n})$.

wild-type animals to be slow growing and paralyzed. ben-1 loss-of-function mutations enable C. elegans to be mobile in the presence of benomyl (Driscoll et al. 1989).

The ben-1 co-conversion strategy was highly efficient in C. elegans experiments (Figure 8A). To develop ben-1 as a co-conversion marker, we used a guide for ben-1 and a single-stranded oligonucleotide HDR template to convert an AA to CTAGAG, thereby creating a new $\mathrm{XbaI}$ site and a premature in-frame translation stop codon downstream (Figure 8A). For measuring co-conversion, we targeted sex-1 with a GG guide and a single-stranded oligonucleotide repair template to insert $11 \mathrm{bp}$ of new sequences, thereby creating stop codons in all three reading frames to prevent translation of the SEX- 1 activation function 2 domain (AF2). We found that $62 \%$ of mobile ben-1 mutant animals had either an imprecise $(37 \%)$ or precise $(25 \%)$ repair event at the sex-1 locus in co-conversion experiments (Figure 8A)

The ben-1 co-conversion strategy was also efficient in $C$. briggsae experiments. We mutated C. briggsae ben-1 using a guide and a single-stranded oligonucleotide HDR template to insert a $\mathrm{T}$ adjacent to the PAM, thereby creating a new NdeI site and a premature in-frame translation stop codon downstream. As in C. elegans, the resulting loss-of-function ben-1 mutations were dominant in C. briggsae, enabling hermaphrodites to be mobile in the presence of benomyl (Figure 8A and Materials and Methods). In co-conversion experiments, we found that $13 \%$ of mobile benomylresistant animals had an HDR event that precisely introduced a $144 \mathrm{bp}$ insert to create a $3 \times O L L A S$ tag in the rpb-1 locus. This result established ben-1 as a good co-conversion marker for C. briggsae.

\section{Selectable co-conversion marker for C. elegans}

To achieve greater ease and efficiency in identifying candidates with genome editing events, we engineered the gene zen-4 to generate a co-conversion marker that would be strongly selectable by permitting reversion of a temperature-sensitive lethal mutant phenotype to wild type. Amino acid substitution D520N in ZEN-4 causes rapid, temperaturesensitive embryonic lethality within $30 \mathrm{~min}$ after zen-4 mutants are shifted from the $15^{\circ}$ permissive temperature to the $25^{\circ}$ nonpermissive temperature (Jantsch-Plunger et al. 2000; Severson et al. 2000). zen-4 (chromosome IV) encodes a member of the kinesin- 6 superfamily of plus-end-directed microtubule motors that is required for polar body extrusion during meiotic divisions, for formation and maintenance of spindle midzone microtubules, and for completion of cytokinesis after mitosis (Powers et al. 1998; Raich et al. 1998; Jantsch-Plunger et al. 2000; Severson et al. 2000; Severson and Bowerman 2002). We used genome editing to create a nematode strain carrying this temperature-sensitive lethal zen-4 allele optimized for use as a co-conversion marker.

The lethal mutant strain zen-4(cle10ts) carried three changes (Figure 9 and Figure S3). The first change was a GAC to AAC transition at codon 520 to create the temperature-sensitive lethal allele. The second was a CGA to CGG synonymous transition at codon 523 to create a PAM without any change in protein sequence. The third was an AluI restriction site introduced by converting codon 519 from GCA to GCT, again with no amino acid change. The singlestranded oligonucleotide repair template would revert zen4(cle10ts) to the wild-type allele, permitting successfully edited animals to be viable at the nonpermissive temperature and hence analyzed for coediting of the primary target gene. Using this strategy, the AluI site would also be eliminated, enabling viable zen-4(+) edited animals to be distinguished from true revertants by a simple PCR assay. The PAM created by the lethal cle10ts mutation would also be lost, thereby preventing the repaired zen-4 allele from being cleaved again by Cas 9 and damaged. This zen-4(ts) selection scheme has an advantage over the selectable co-conversion scheme using a pha-1(ts) lethal mutation in not leaving a SNP behind in the genome (Ward 2015).

We tested the efficiency of zen-4 as a co-conversion marker by comparing the frequency of inserting DNA encoding a hemagglutinin (HA) tag into the lir-2 gene using zen-4 or dpy-10 as a co-conversion marker. Of viable zen-4(+) animals, 39\% had the ha::lir-2 insertion, and 44\% had imprecise repair at lir-2 (Figure 9A). The $83 \%$ overall success rate at lir2 was more favorable than the $65 \%$ rate with $d p y-10$ as the co-conversion marker (30\% with the ha::lir-2 insertion and $35 \%$ with imprecise repair). This result suggested that zen-4 editing is less efficient than $d p y$-10 editing, causing the relative frequency of edits at desired loci to be higher in zen-4(+) animals than in $d p y-10$ mutants. This hypothesis was validated by our result that only $33 \%$ of $d p y-10$ mutants were converted to zen-4(+) (Figure 9B). Decreased editing 


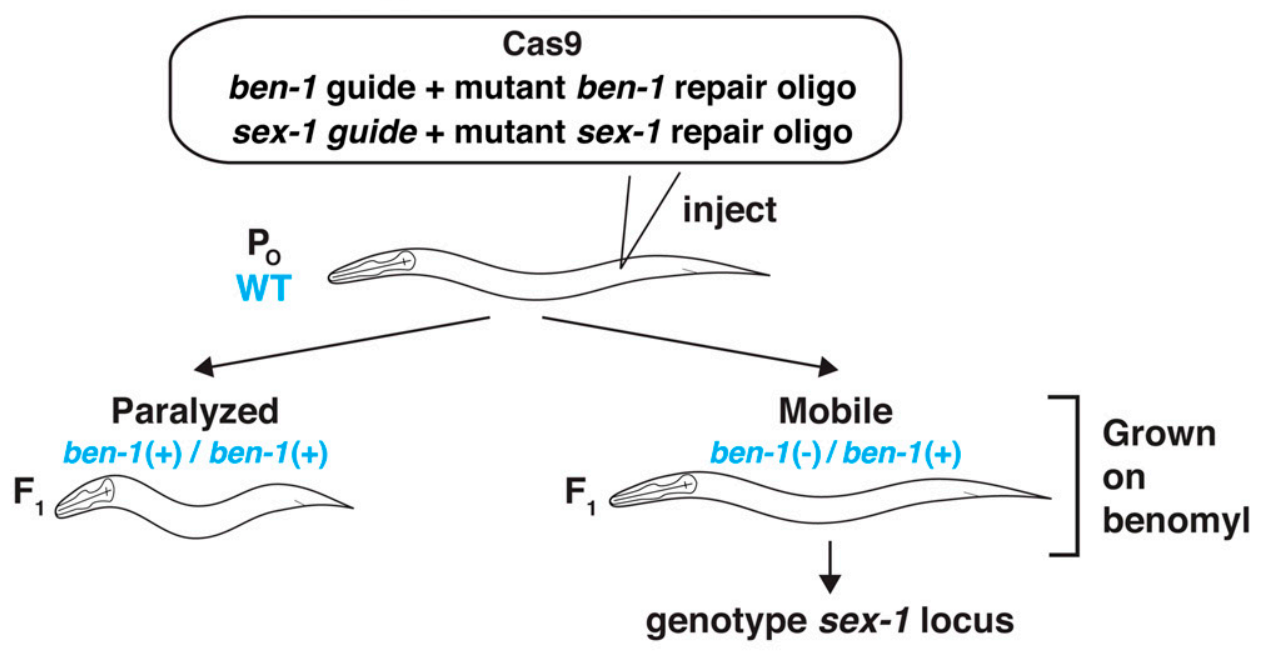

\section{Ce-ben-1(+) paralyzed}

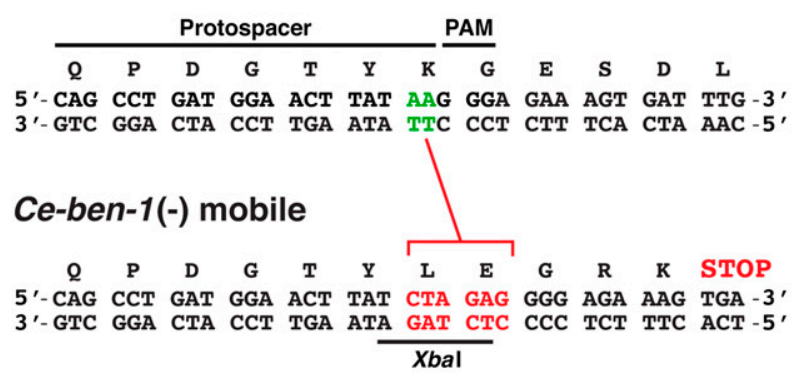

B

ben-1 co-conversion marker for $C$. briggsae

\section{Cbr-ben-1(+) paralyzed}

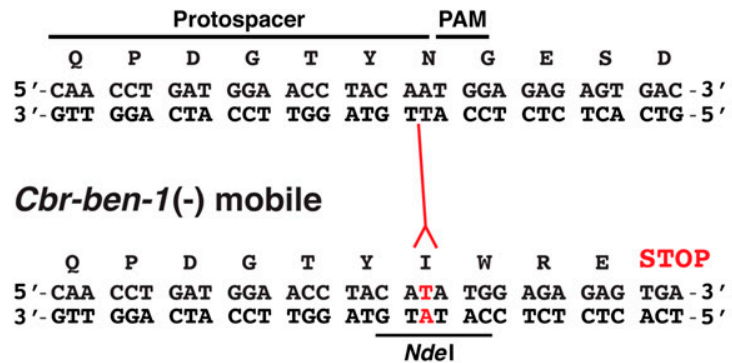

Ce-sex-1

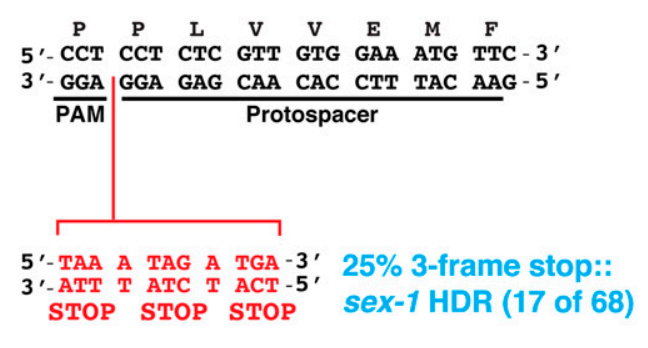

Also, $37 \%$ sex-1 imprecise repair (25 of 68 )

\section{Cbr-rpb-1}

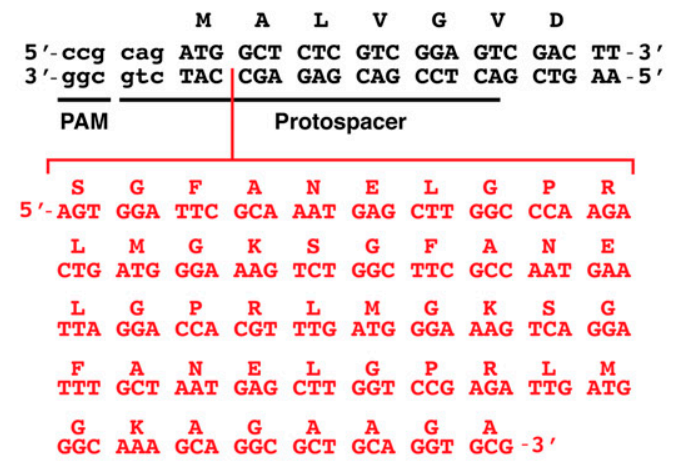

13\% 3xOLLAS::rpb-1 HDR (12 of 95)

Figure 8 Efficient co-conversion marker to edit genomes of diverged nematode species. Mutations in the highly conserved gene ben-1, which encodes $\beta$-tubulin, confer resistance to benomyl, a drug that binds to $\beta$-tubulin, inhibits microtubule polymerization, and causes genetically wild-type animals to be slow growing and paralyzed. Loss-of-function mutations in ben-1 confer dominant benomyl resistance in both C. elegans and C. briggsae. (A) In C. elegans, the repair template created an Xbal restriction site adjacent to the PAM by converting an AA to CTAGAG, resulting in an in-frame stop codon that caused premature translation termination. (B) In C. briggsae, the repair template created an Ndel site by inserting a T adjacent to the PAM, causing an in-frame translation termination stop codon. In both species, the repair template altered protospacer sequences adjacent to the PAM, preventing the ben-1 mutants from being cleaved by Cas9. Successful editing of $C$. elegans sex-1 and C. briggsae rpb-1 established ben-1 as an effective co-conversion marker. WT, wild type. 


\section{A zen-4 co-conversion marker for $C$. elegans}

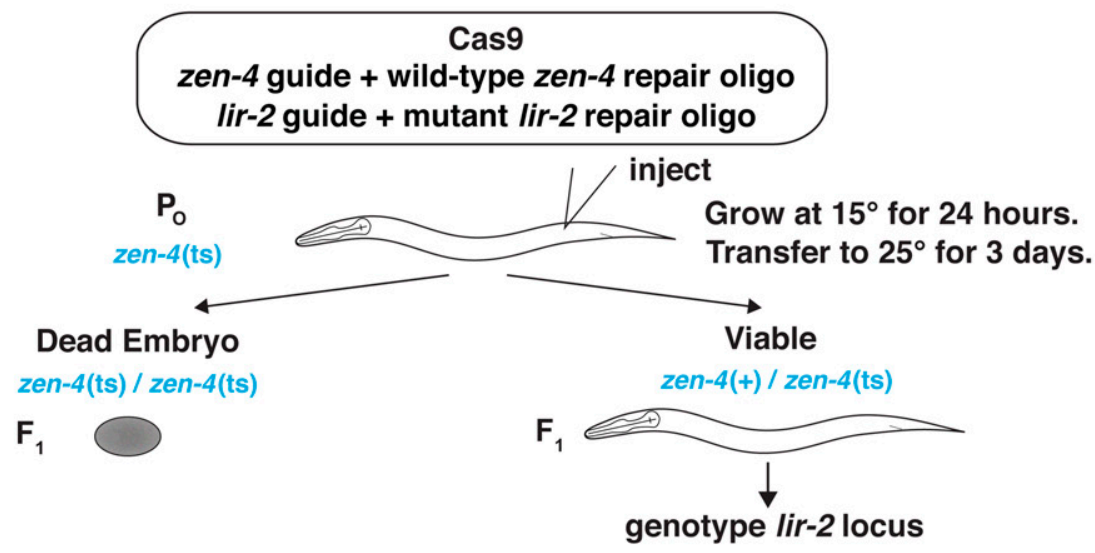

zen-4(cle10 ts) IV dead at $25^{\circ} \mathrm{C}$

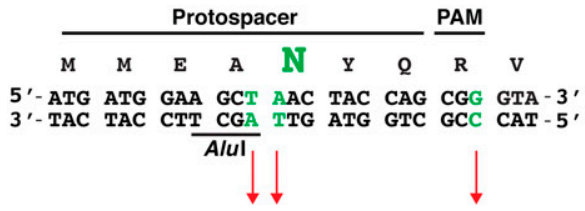

zen-4(+) alive at $25^{\circ} \mathrm{C}$

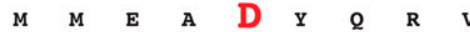

5'-ATG ATG GAA GCA GAC TAC CAG CGA GTA-3

3'-TAC TAC CTT CGT CTG ATG GTC GCT CAT-5, ha::lir-2 II

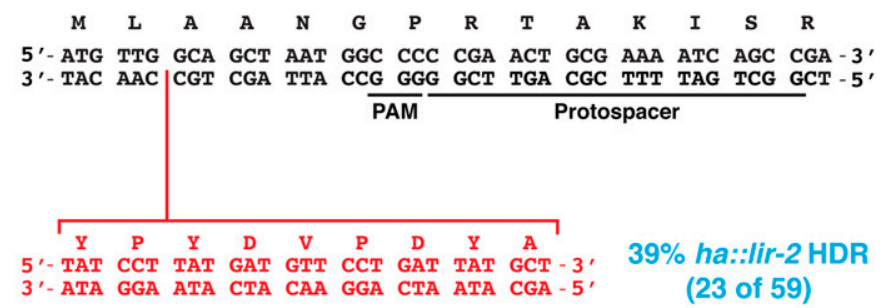

Also, $44 \%$ lir-2 imprecise repair (26 of 59 )
(23 of 59$)$

\section{Comparison with $d p y-10(\mathrm{gf})$ as a co-conversion marker}

\section{dpy-10(+) II}

$$
\begin{array}{llllllllllllll}
T & \text { A } & \mathbf{R} & \text { G } & \text { A } & \text { Y } & \text { G } & \text { S } & \text { G } & \text { A }
\end{array}
$$

5'-ACC GCT CGT GGT GCC TAT GGT AGC GGA GCT-3'

3'- TGG CGA GCA CCA CGG ATA CCA TCG CCT CGA-5'

$$
\overline{\text { PAM }}
$$

$$
\text { Protospacer }
$$

dpy-10(gf)

$\begin{array}{lllllllllll} & T & A & C & G & A & Y & G & S & G & A\end{array}$

5'- ACC GCA TGC GGT GCC TAT GGT AGC GGA GCT-3'

3'- TGG CGT ACG CCA CGG ATA CCA TCG CCT CGA-5'

$$
\text { Sphl }
$$

ha::lir-2 II

30\% ha::lir-2 HDR

(27 of 91)

Also, 35\% lir-2 imprecise repair

(32 of 91 )

\section{B Examples of zen-4 as a co-conversion marker}

dpy-10(+); zen-4(cle10 ts) injected strain

Co-conversion

marker

dpy-10(gf) II

zen-4(+) IV

zen-4(+) IV

\begin{tabular}{l} 
Outcome \\
\hline$z e n-4(+) I V$ \\
$d p y-10(g f) ~ I I$ \\
$3 x f l a g:: c o h-4 V$
\end{tabular}

Co-conversion HDR efficiency

$33 \% \quad(8$ of 24$)$

$74 \%$ (28 of 38$)$

$60 \%(19$ of 32$)$

Figure 9 Selectable co-conversion marker for C. elegans. (A) zen-4(+) produces an essential plus-end-directed microtubule motor and acts as an efficient co-conversion marker to revert the rapid, temperature-sensitive lethality caused by a defective ZEN-4 protein with amino acid substitution D520N produced by zen-4(cle10ts) mutants. Viable zen-4(+) progeny are scored for editing in genes of choice. (B) Successful editing of lir-2, dpy-10, and coh-4 established zen-4 as an effective co-conversion marker. Comparison of editing efficiency using $d p y-10$ and zen-4 as co-conversion markers is also presented. 
efficiency at zen-4 is likely due to the need for precise HDR to yield a wild-type zen-4(+) phenotype, but both precise and imprecise repair at $d p y-10$ yield obvious phenotypes. The decreased editing efficiency and the strong selection for viability make zen-4 a valuable co-conversion marker.

zen-4 was highly successful as a co-conversion marker for editing other loci (Figure 9B). For example, $60 \%$ of zen-4(+) edited animals acquired a DNA insertion encoding a FLAG tag in the cohesin subunit gene coh-4. Also, 74\% of zen-4(+) edited animals acquired a $d p y-10$ mutation.

\section{Efficient genome editing occurs in embryos}

Thus far, we have evaluated factors that influence the success of genome editing in self-fertile hermaphrodites. In these hermaphrodites, Cas9 RNPs and repair templates were introduced into the gonad syncytium of young adults that had already produced a full complement of sperm but still had hundreds of prophase nuclei destined to become cellularized and mature into oocytes. For fertilization to occur, the Cas9exposed oocytes had to pass through the spermatheca, the organ that stores mature ameboid spermatozoa derived from either self-fertile hermaphrodites or males during male/hermaphrodite mating. In principle, Cas9-induced cleavage and repair events could occur at any time during meiotic prophase or later during embryogenesis, either before or after oocyte and sperm pronuclei had formed and fused. To distinguish between these possibilities, we launched a series of experiments to evaluate (1) the frequency and type (precise $v s$. imprecise) of DNA editing in embryos $v s$. that in meiotic prophase, (2) the efficiency of editing genomes from male sperm $v s$. genomes from hermaphrodite sperm or oocytes, and (3) the effect of copulation per se on genome editing.

We first addressed whether differences in editing efficiency were detectable between progeny from self-fertile hermaphrodites $v s$. progeny from hermaphrodites mated for $24 \mathrm{hr}$ by wild-type males (Figure 10, A and B). Using $d p y-10$ as a co-conversion marker, we found that the frequency of indels in the atf-2 II gene of $d p y-10$ mutants rose to almost twice the level in the outcrossed progeny of mated hermaphrodites (61\%) vs. the self-progeny of unmated hermaphrodites (35\%) $(P=0.001$, chi square).

In subsequent experiments, we specifically targeted either the paternal allele (male sperm genome) or maternal allele (hermaphrodite oocyte genome) of a locus in mated hermaphrodites. In principle, because Cas9 has the opportunity to cleave hermaphrodite oocyte DNA both in developing germ cells and in embryos, the efficiency of editing maternal DNA might be expected to exceed the efficiency of editing paternal DNA, which must occur only after fertilization in these experiments. We found the opposite, consistent with the mating experiment above.

A Cas9-targetable polymorphic allele of a locus was introduced into the embryo from either the hermaphrodite oocyte or male sperm. This allele had a SNP in the penultimate position of the target-specific DNA sequence within the spacer seed sequence, permitting allele-specific Cas9 cleavage without off-target cleavage of wild-type alleles (Materials and Methods). For the gene sex-1, when the targetable polymorphic AG allele was contributed from the male sperm, an average of $88 \%( \pm 2)$ of $d p y-10$ mutants had an indel in the paternal allele of sex-1 (Figure 10C). In contrast, when the targetable AG allele was contributed from the hermaphrodite oocyte, an average of $43 \%( \pm 4)$ of $d p y-10$ mutants had an indel in the maternal sex-1 allele (Figure 10D) $\left(P<10^{-5}\right.$, chi square). The rate of mutagenesis for the paternal AG allele was also twice that for the identical AG alleles in homozygous self-fertile hermaphrodites $(43 \% \pm 6)\left(P<10^{-5}\right.$, chi square), even though the male sperm genome had half the number of targetable alleles as self-fertile hermaphrodites, and hermaphrodite alleles could be edited in both germ cells and embryos, while the paternal allele could only be edited in the embryo (Figure 10E).

We conclude that (1) highly efficient genome editing does indeed occur in embryos, (2) male sperm DNA can be targeted more effectively than hermaphrodite oocyte or sperm DNA, and (3) the act of mating per se does not increase the efficiency of genome editing. Our discovery of homozygous identical indels in the progeny of self-fertile hermaphrodites supports the conclusion that genome editing is efficient in embryos after pronuclear fusion (Figure 10, B, E, and H). Homozygous indels arose from Cas9 cleavage of the unmutagenized parental genome in the embryo followed by HDR using a homologous chromosome with an indel for the template. Even when these homozygous indels (Figure 10E) were counted as independent Cas9 events and added to those causing the original heterozygous indels, the total frequency of Cas9-mediated repair in hermaphrodite sperm and oocyte genomes (59\%) was less than the frequency in paternal genomes of cross-progeny embryos $(88 \% ; P=0.002$, chi square). Furthermore, a larger number of independent, distinct indels were formed in male sperm genomes than in hermaphrodite sperm and oocyte genomes, indicating that male sperm genomes were targeted more effectively than genomes of hermaphrodite gametes. In mouse embryos, editing of sperm genomes also occurred more efficiently than editing of egg genomes (Suzuki et al. 2014).

These findings with sex-1 were confirmed at two other loci, atf-2 II (Figure 10, F-H) and an intergenic locus on chromosome I (Figure S5A). At atf-2, for example, $90 \%$ of $d p y-10$ mutants had indels in male sperm alleles compared to $35 \%$ of dpy-10 mutants with indels in hermaphrodite oocyte alleles $\left(P<10^{-5}\right.$, chi square) or $31 \%$ of $d p y-10$ mutants with indels in hermaphrodite sperm or oocyte alleles $\left(P<10^{-5}\right.$, chi square) (Figure 10, F-H). The findings were also reproduced in a second set of sex-1 experiments in which the wild-type GG allele was targeted rather than the polymorphic AG allele (Figure S5B).

While highly elevated rates of editing were observed for male sperm DNA at inter- and intragenic sites on three different chromosomes, an exception occurred at the lir-2 locus (Figure S5C). The paternal allele was targeted at half the rate $(36 \%)$ as alleles in self-fertile hermaphrodites 
(72\%), revealing that mating can improve editing rates at many, but not all, loci.

\section{Precise DSB repair via inter-homolog HDR occurs at high frequency in embryos}

Use of polymorphic alleles to restrict editing to either the paternal or the maternal genome enabled us to make two further comparisons. We assessed the efficiency of recovering mutations in the target gene when both the co-conversion marker and the target gene were cleaved and repaired in the same parental genome (either maternal or paternal) or instead in different genomes (Figure 11). We also compared the frequency of precise DSB repair in embryos via HDR from the homologous chromosome (inter-homolog HDR) to the frequency of imprecise repair via indel formation (Figure 11). The design of experiments in Figure 11 allowed us to quantify bona fide interhomolog HDR events in embryos. This class of repair events had been inferred from the recovery of identical homozygous indels in prior experiments (Figure 10, B, E, and $\mathrm{H}$ ).

For the first comparison, the success in obtaining precise or imprecise mutations in sex- 1 was invariably higher if both the marker and gene were targeted in the same parental genome, either paternal or maternal ( $P<10^{-5}$, chi square). When paternal alleles for both the marker and sex-1 were targeted, $92 \%( \pm 2)$ of $d p y-10$ mutants had a mutation (precise or imprecise) in the paternal sex-1 allele (Figure 11A). In contrast, only $41 \%$ ( \pm 9 ) of $d p y-10$ mutants had a mutation in the paternal sex-1 allele when the maternal $d p y$-10 allele was targeted $\left(P<10^{-4}\right.$, chi square) (Figure 11C). Similarly, when maternal alleles were targeted for both the marker and sex-1, 79\% ( \pm 5$)$ of $d p y-10$ mutants had a mutation in the maternal sex-1 allele (Figure 11B). However, only $30 \%$ $( \pm 8)$ of $d p y-10$ animals had a mutation in the maternal sex-1 allele when the paternal $d p y-10$ allele was targeted $(P<5 \times$ $10^{-4}$, chi square) (Figure 11D).

For the second comparison, a surprisingly high fraction of DSB repair events occurred precisely via inter-homolog HDR (0.26-0.57 of total sex-1 mutations) when either the maternal or paternal sex-1 locus was targeted for cleavage and repair (Figure 11, A-D). This result indicates that HDR occurs at high frequency in embryos and that the repair must have taken place after fusion of the paternal sperm and maternal oocyte pronuclei, when homologs were in proximity. Repair of Cas9 DSBs through inter-homolog HDR has also been observed in mice and tomatoes (Wu et al. 2013; Filler Hayut et al. 2017; Ma et al. 2017).

\section{Precise HDR from an exogenous template occurs at high frequency in embryos}

The high frequency of inter-homolog HDR events in crossprogeny embryos (Figure 11) raised the question of whether the frequency of HDR using exogenous templates would also be high in cross-progeny embryos. We determined the frequency of HDR from exogenous templates and compared it directly with that of inter-homolog HDR and imprecise repair (Figure 12, A and B). When only paternal alleles of both sex-1 and $d p y-10$ were targeted in cross-progeny embryos, HDR events from exogenous templates occurred at a good frequency (24\% of all sex-1 mutations), and they were correlated with a reduction of both inter-homolog HDR events (reduced from 33 to $23 \% ; P<10^{-3}$, chi square) and imprecise repair (reduced from 67 to $53 \% ; P<10^{-5}$, chi square) (Figure 12A). Because paternal alleles can only undergo repair in embryos, and each form of repair precludes the others, all forms are predicted to be in direct competition, as observed in our data.

When only maternal alleles of both sex-1 and $d p y$-10 were targeted in cross-progeny embryos, HDR events from exogenous templates also occurred at good frequency (30\% of all sex-1 mutations), one not statistically different from related HDR events at paternal alleles $(P=0.2)$ (Figure 12B). However, in contrast to HDR events with exogenous templates at paternal alleles, these HDR events at maternal alleles correlated with a loss of imprecise repair (reduced from 90 to $56 \%$; $P<10^{-5}$, chi square), but not a loss of inter-homolog HDR (changed from 10 to $14 \% ; P=0.2$, chi square). These results suggest that, for maternal alleles, imprecise repair and HDR using exogenous templates occur in a similar window of time, during meiotic prophase or until pronuclear fusion in embryos, but inter-homolog HDR occurs later. Both the insignificant change in frequency of inter-homolog HDR events when exogenous HDR templates were present and the general low frequency of inter-homolog HDR events are consistent with inter-homolog HDR occurring after pronuclear fusion in embryos, once other forms of repair had occurred.

We also asked whether HDR from exogenous templates occurs in embryos of self-fertile hermaphrodites. We found that $19 \%$ of total sex-1 mutants had an HDR event from an

nontargetable polymorphic alleles are shown in gray. The far-left column shows the configuration of oocyte and sperm alleles [maternal ( $\mathrm{m}$ ) or paternal (p)] for the $d p y-10$ co-conversion marker and sex-1 or atf-2 in mated or self-fertile hermaphrodites. The ovals display the genotypes of sex-1 or atf-2 loci in Dpy or Rol progeny of mated (blue) or self-fertile (red) hermaphrodites as determined by sequence analysis. * indicates the polymorphic allele with an indel. This allele is shown in parenthesis because the indel occasionally changes the allele-specific sequence. For self-fertile hermaphrodites in B, $\mathrm{E}$, and $\mathrm{H}$ as well as for mated hermaphrodites in A, either the sperm or oocyte allele could be repaired imprecisely to form an indel. Thus, only one arbitrary allele is shown with an indel. For C-E the average percentage of indels \pm SEM is provided in blue (mated hermaphrodites) or red (self-fertile hermaphrodites), and the percentages for individual replicates are shown in black. The numbers in brackets below the percentage of indels show the fractions of total indel-laden progeny that have identical homozygous indels. For mated hermaphrodites, the percentage of sex-1 or atf-2 indels within Dpy or Rol outcrossed progeny $(n)$ was calculated by the formula: (number of indels in target gene)/(total number of Rol or Dpy $F_{1}$ outcrossed progeny) $\times 100$. For self-fertile hermaphrodites, the percentage of sex-1 or atf-2 indels within total Dpy or Rol progeny ( $n$ ) was calculated by the formula: (number of indels in target gene)/(total number of Rol or Dpy $F_{1}$ s in self-progeny) $\times 100$. 
$\%$ of Total Rol and/or Dpy F1s

(n) with Mutations in sex-1
Indels /

Total sex-1 Mutations

Indel /

Rol and/or Dpy

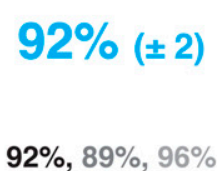

92\%, 89\%, 96\%

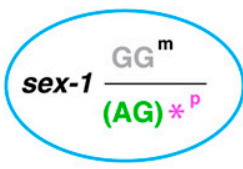

0.57
A Target Paternal sex-1 and dpy-10

$$
\begin{array}{cc}
\frac{\text { oocyte }}{\operatorname{sex}-1 \mathrm{GG}^{\mathrm{m}}} & \frac{\text { sperm }^{\mathrm{m}}}{\operatorname{sex}-1 \underline{\mathrm{AG}^{\mathrm{p}}}} \\
\text { dpy-10 } \underline{\mathrm{GG}} & \text { dpy-10 } \underline{\mathrm{AG}^{p}}
\end{array}
$$

Target Maternal sex-1 and dpy-10

$\begin{array}{cc}\frac{\sigma^{\prime} \text { sperm }}{\operatorname{sex}-1 \underline{\mathrm{AG}^{\mathrm{m}}}} & \frac{\operatorname{sex}-1 \underline{\mathrm{GG}^{\mathrm{p}}}}{d p y-10 \underline{\mathrm{AG}^{\mathrm{m}}}} \\ d p y-10 \underline{\mathrm{GG}^{p}}\end{array}$

$\mathbf{0 . 6 0}, 0.61,0.42$

$68 / 131$

$$
79 \%( \pm 5)
$$

$74 \%, 84 \%$

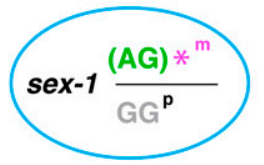

0.74
Inter-homolog HDR /

Total sex-1 Mutations

Inter-homolog HDR /

Rol and/or Dpy

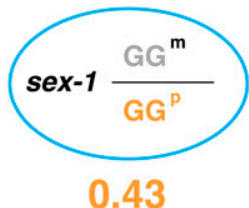

$\mathbf{0 . 4 0 , 0 . 3 9 , 0 . 5 8}$

$52 / 131$

$\mathbf{0 . 7 2}, 0.76$

$39 / 68$

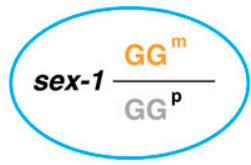

0.26

$\mathbf{0 . 2 8}, 0.24$

\begin{tabular}{|c|c|c|}
\hline oocyte & orsperm & \\
\hline $\operatorname{sex}-1 \mathrm{GG}^{\mathrm{m}}$ & $\operatorname{sex}-1 A^{p}{ }^{p}$ & $41 \%( \pm 9)$ \\
\hline$d p y-10 \underline{A G^{m}}$ & dpy-10 $\mathrm{GG}^{\mathrm{p}}$ & \\
\hline
\end{tabular}

C Target Paternal sex-1 and Maternal dpy-10

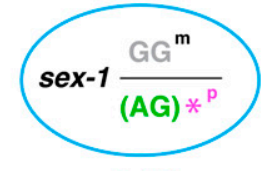

0.43

$\mathbf{0 . 4 4}, 0.33,0.56$

$29 / 155$

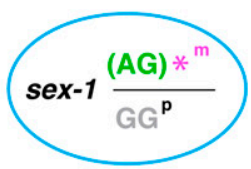

0.43

0.33, 0.45

$17 / 124$

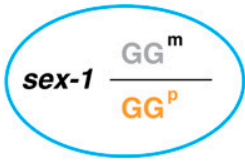

0.57

$\mathbf{0 . 5 6}, 0.67,0.44$

$38 / 155$

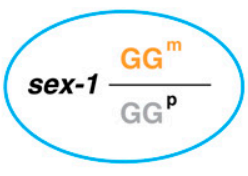

0.57

$\mathbf{0 . 6 7}, 0.55$

Figure 11 Efficiency of recovering genome editing events is greater if the co-conversion marker and target gene are cleaved and repaired in the same parental genome. Comparison is shown of total sex-1 mutation frequency (indels and inter-homolog HDR events) when the dpy-10 co-conversion marker was targeted ( $A$ and $B$ ) in the same or (C and $D)$ in different parental genomes [either paternal $(p)$ or maternal $(m)$ ] as sex-1. Comparison is also shown for the frequency of imprecise DNA repair events via indel formation vs. the frequency of precise DNA repair in embryos via inter-homolog HDR. Cas9targetable polymorphic alleles of loci are shown in green and nontargetable polymorphic alleles are shown in gray. The far-left column shows the configuration of hermaphrodite oocyte $(\mathrm{m})$ and male sperm $(\mathrm{p})$ alleles for $d p y-10$ and sex-1. Frequency of total sex-1 mutations relative to the total number (n) of Rol or Dpy cross-progeny was calculated by the formula: (total number of sex-1 indels and inter-homolog HDR events)/(total number of Rol or Dpy $F_{1}$ s) $\times 100$. The average of percentages for all replicates of total sex-1 mutations \pm SEM are shown in blue and the percentages for individual replicates are shown in black, medium gray, and light gray. Frequency in obtaining mutations in sex-1 (combination of indels and inter-homolog HDR events) was invariably higher if both the marker and target gene were cleaved and repaired in the same parental genome, either paternal or maternal $\left(P<10^{-5}\right.$, chi square). The left column of ovals shows the genotypes of progeny with sex-1 indels in Dpy or Rol outcrossed progeny, as determined by sequence analysis. * indicates the polymorphic allele with an indel. This allele is shown in parenthesis because the indel occasionally changes the AG sequence. The fraction of sex-1 indels included in the total number of sex-1 mutations (indels and inter-homolog HDR events) for all replicates combined is shown in red. The total number of indels compared to the total number of sex-1 mutations for all replicates combined is shown in brown. The right column of ovals shows the genotypes of sex-1 inter-homolog HDR events in Dpy or Rol outcrossed progeny, as determined by sequence analysis. The fraction of sex-1 inter-homolog HDR events included in the total number of sex-1 mutations for all replicates combined is shown in orange. The respective replicates for fractions of indels and inter-homolog HDR events are shown black, medium gray, and light gray, matched to replicates for total percentages of sex-1 mutations in each experiment. The total number of inter-homolog HDR events compared to the total number of sex-1 mutations for all replicates combined is shown in brown. Remarkably, precise DSB repair via inter-homolog HDR, like indel formation, occurs at high frequency in embryos from mated hermaphrodites. In C, quantification of inter-homolog events was based on the assumption that $100 \%$ of the progeny were cross-progeny. The validity of this assumption was based on our observation that all Dpy or Rol progeny in A, B, and D were cross-progeny, as determined by the criteria given in Materials and Methods. 


\section{$\%$ of Total Rol and/or \\ Dpy F1s (n) with}

Mutations in sex-1

A Target Paternal sex-1 and $d p y-10$

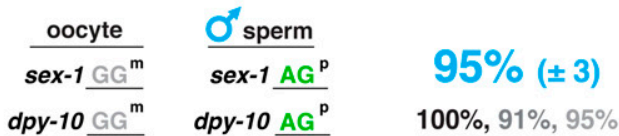

no HDR template
Indels / Total
sex-1 Mutations

Indel /

Rol and/or Dpy

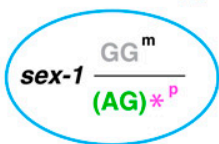

0.67

$\mathbf{0 . 5 6}, 0.69,0.77$

$169 / 265$

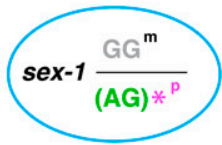

0.53

$\mathbf{0 . 5 0}, 0.64,0.46$
Inter-homolog HDR / Exogenous template

Total sex-1 Mutations HDR / Total

sex-1 Mutations

Inter-homolog HDR / Exogenous template Rol and/or Dpy HDR/Rol and/or Dpy

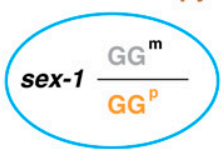

0.33

$\mathbf{0 . 4 4}, 0.31,0.23$

$84 / 265$

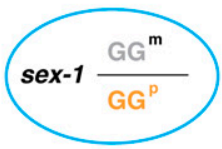

0.23

$\mathbf{0 . 4 3}, 0.31,0.23$

$47 / 267$

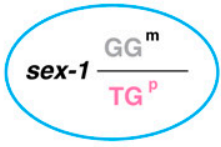

0.24

$\mathbf{0 . 2 5}, 0.21,0.26$

$50 / 267$

B Target Maternal sex-1 and dpy-10

$112 / 267$

\begin{tabular}{|c|c|c|}
\hline oocyte & orperm & \\
\hline sex-1 $\underline{A G}^{m}$ & $\operatorname{sex}-1 G^{p}$ & $\%( \pm 4)$ \\
\hline py-10 $\underline{A G}^{m}$ & $d p y-10 \mathrm{GG}^{\mathrm{p}}$ & $74 \%$ \\
\hline
\end{tabular}

no HDR template

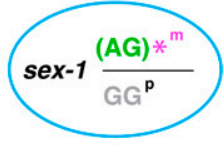

0.90

$0.91,1.00,0.82$

$170 / 256$

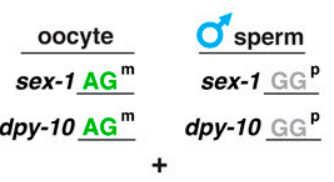

sex-1 HDR template 5 '
$76 \%( \pm 3)$

$77 \%, 71 \%, 80 \%$

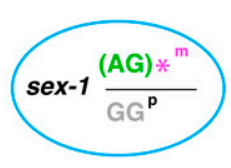

0.56

$0.67,0.48,0.52$

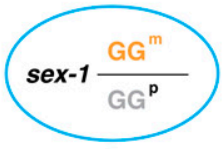

0.10

$0.09,0.00,0.18$

$19 / 256$

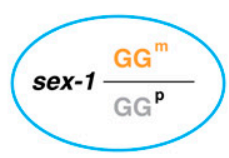

0.14

$\mathbf{0 . 1 6}, 0.08,0.18$

$27 / 260$

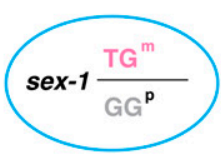

0.30

$\mathbf{0 . 1 8}, 0.44,0.30$

\section{Target ${ }^{\prime}$ Oocyte and Sperm sex-1 and dpy-10}

$$
\begin{aligned}
& \text { oocyte } \\
& \operatorname{sex}-1{A G^{m}}^{m} \\
& \text { dpy-10 } \mathrm{AG}^{\mathrm{m}} \\
& \frac{{ }^{\prime} \text { sperm }}{\text { sex-1 }{ }^{A G^{m}}} \\
& \text { dpy-10 } \underline{A G}^{m}
\end{aligned}
$$

no HDR template

$$
\begin{aligned}
& \frac{\text { oocyte }}{\text { sex-1 } \underline{A G}^{\mathrm{m}}} \quad \frac{\Phi^{\prime} \text { sperm }}{{\text { sex-1 }{ }^{A G}}^{\mathrm{m}}} \\
& \text { dpy-10 } \underline{\mathrm{AG}^{\mathrm{m}}} \quad \text { dpy-10 } \underline{\mathrm{AG}}^{\mathrm{m}} \\
& + \\
& \text { sex-1 HDR template 5' }
\end{aligned}
$$
$66 \%, 88 \%, 70 \%$

† sex-1 and atf-2 mutations could be in oocyte or sperm allele, but generally not both.

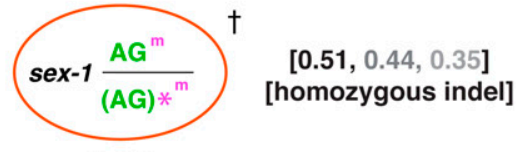

1.00

$148 / 271$

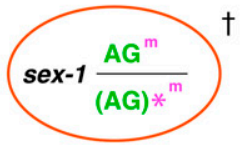

$\mathbf{0 . 8 1}$

$\mathbf{0 . 9 7}, 0.75,0.70$

$161 / 272$

[0.21, 0.06, 0.03]

[homozygous indel]

Figure $12 \mathrm{HDR}$ from an exogenous repair template occurs at high frequency in embryos. (A and B) Comparison is shown for total sex-1 mutation frequency (blue), including either indels and precise inter-homolog HDR events (top) or indels, precise inter-homolog HDR events, and precise HDR from an exogenous single-stranded template (bottom) when the $d p y-10$ co-conversion marker was targeted in mated hermaphrodites in the same parental genomes as sex-1, either paternal $(p)$ in A or maternal $(m)$ in B. The 100 nt single-stranded HDR template had only a single nucleotide change from the wild-type sequence. The AG sequence immediately $5^{\prime}$ of the PAM in the protospacer strand was changed to TG. Comparison is also shown for the fraction of indels (black, left column of ovals), the fraction of precise inter-homolog HDR events (orange, middle column of ovals), and the fraction of precise HDR events from the exogenous repair template (pink, right column of ovals). The replicates for each category are shown in black, medium gray, and light gray. The configurations of hermaphrodite oocyte $(\mathrm{m})$ and male sperm ( $\mathrm{p}$ ) alleles for $d p y-10$ and sex-1 are displayed as in the left column of 
exogenous template (Figure 12C, bottom). Moreover, the total frequency of $d p y-10$ co-converted animals having a sex-1 mutation rose from $55 \%( \pm 4)$, when only heterozygous indels were scored in experiments lacking a homologous repair template (Figure 12C, top), to 74\% ( \pm 6$)$, when both indels and HDR events were scored in experiments with a homologous repair template $\left(P<10^{-5}\right.$, chi square) (Figure $12 \mathrm{C}$, bottom). This latter result revealed that quantifying only heterozygous indels in progeny of self-fertile hermaphrodites did not reflect the total frequency of independent editing events. Indeed, inclusion of inter-homolog HDR events deduced from the occurrence of homozygous identical indels in experiments lacking an exogenous repair template increased the total frequency of sex-1 editing events from 55 to $78 \%$, (see calculation in Figure 12C legend), a frequency not different from the $74 \%$ found when including HDR events from exogenous templates.

To infer where HDR via exogenous templates had occurred, we compared the proportion of heterozygous indels and homozygous indels in progeny of self-fertile hermaphrodites that had been given Cas9 RNPs either with or without an exogenous HDR template. We found that HDR from an exogenous template correlated with a reduction in the fraction of homozygous identical indels ( $P<10^{-5}$, chi square) but not a reduction in the fraction of unique heterozygous indels $(P=$ 0.3 , chi square). Thus, HDR from an exogenous template appeared to have competed with inter-homolog HDR, which can only occur in embryos after fusion of oocyte and sperm pronuclei. It did not compete with imprecise repair, which can occur during meiotic prophase or in embryos. We infer that genomes in embryos from self-fertile hermaphrodites undergo a substantial number of HDR events from exogenous templates, most likely after pronuclear fusion.

\section{Conclusions}

Our Cas9-editing strategies have achieved highly efficient genome engineering in $C$. elegans. These innovations can be exploited to enhance genome editing in diverse species.

First, our detailed analysis of imprecise repair outcomes in C. elegans, along with data from other species (Mali et al. 2013b; van Overbeek et al. 2016; Richardson et al. 2018), showed that nontemplated repair at Cas9 cleavage sites is asymmetric, favoring deletions and insertions $5^{\prime}$ of the PAM on the protospacer strand. Thus, imprecise repair can be increased significantly by selecting Cas9 targets with PAMs located 3' of desired changes.

Second, our systematic analysis also showed that HDR from a single-stranded repair template is directional and consistent with an SDSA mechanism. We exploited this finding to devise guidelines for inserting precise changes with high frequency into sites within close proximity (30 bp) to the DSB. Insertions $5^{\prime}$ of the PAM can be achieved efficiently using a single-stranded repair template corresponding to the protospacer. In contrast, precise changes $3^{\prime}$ of the PAM are achieved most reliably using a spacer strand repair template instead. Both spacer and protospacer strand templates are effective for inserting precise changes directly at the DSB site. Because the bound Cas9 nickase RuvC also has a $3^{\prime}-5^{\prime}$ exonucleolytic activity that catalyzes limited resection of the cleaved protospacer strand, a spacer strand repair template can elicit not only high-efficiency insertion of polymorphisms $3^{\prime}$ of the PAM but also low-efficiency insertion of polymorphisms just $5^{\prime}$ of the PAM.

Third, we overcame limitations related to inserting long non-homologous fragments of DNA near a DSB and to engineering small specific changes at considerable distance from a DSB. These desired outcomes had been problematic because HDR from a double-stranded repair template is inefficient, distance dependent, and highly directional, favoring changes $5^{\prime}$ of the PAM. The strategy that surmounted these limitations uses two different Cas9 guides to create two DSBs that flank the region intended for insertion of exogenous DNA. This approach is effective for inserting $\sim 10 \mathrm{~kb}$ of non-homologous DNA and for incorporating a series of nucleotide substitutions along the entire length of a region, up to $1.5 \mathrm{~kb}$ from a DSB. The scheme is also beneficial for editing insertion sites such as AT-rich introns that are not adjacent to DNA sequences necessary for effective Cas9 guide design.

Use of two Cas 9 targets required that we determine the orientation of PAMs most successful for editing. One

Figure 11, A and B. Genotypes of progeny with indels and inter-homolog HDR events are displayed like the right and left ovals of Figure 11, A and B. The formula for calculations and method of displaying replicates is the same as in Figure 11, A and B. The right column of ovals shows the genotypes of progeny with precise HDR from the exogenous repair template, as determined by sequence analysis. Shown in brown for each column of ovals is either the total number of sex-1 indels found in all Rol and/or Dpy progeny from all replicates, the total number of sex-1 inter-homology HDR events found in all Rol and/or Dpy progeny from all replicates, or the total number of sex-1 HDR events via exogenous templates found in all Rol and/or Dpy progeny from all replicates. These experiments reveal that HDR using exogenous templates occurs in cross-progeny embryos from male/hermaphrodite matings when either the maternal (oocyte) or paternal (sperm) allele is targeted for Cas9-mediated DNA cleavage. (C) Comparison is shown among progeny of self-fertile hermaphrodites of the frequency (red) of imprecise DNA repair via indel formation (top) vs. the frequency (red) of imprecise DNA repair (indels) and precise DNA repair via HDR from the exogenous $100 \mathrm{nt}$ single-stranded template (bottom). The genotypes of parents and progeny are displayed as in Figure 10E. Experimental replicates showing the fraction of indels (top) and fraction of indels and HDR events (bottom) are displayed in black, medium gray, and light gray. Numbers in brackets represent the fraction of total indel-laden progeny that have identical homozygous indels. Shown in brown is the total number of sex-1 indels or the total number of sex-1 HDR events from exogenous templates found in all Rol and/or Dpy mutants from all combined replicates. The total frequency of Cas9-editing events reported in the text, when taking into account homozygous identical indels as independent Cas 9 cleavage events, was calculated by the formula: [(fraction of total Rol and/or Dpy $F_{1} s$ with mutations in sex-1) + (fraction of total Rol and/or Dpy $F_{1} s$ with homozygous identical indels in sex-1)] $\times 100$. These experiments reveal that genomes of embryos from self-fertile hermaphrodites undergo a substantial number of HDR events from exogenous templates. * indicates the polymorphic allele with an indel. This allele is shown in parenthesis because the indel occasionally changes the AG sequence. 
orientation causes the PAM to remain on the chromosome end after DNA excision; the other causes the PAM to reside on the DNA sequence excised from the chromosome. Editing is most efficient if both PAMs will remain on chromosome ends after DNA excision.

Fourth, we optimized Cas9 delivery methods and guide RNA design. We assessed the relative effectiveness of using either pre-assembled RNP complexes of guide RNAs and Cas9 or DNA expression vectors to deliver editing reagents to gonads. Using both delivery methods, we also assessed the effectiveness of guide RNAs differing only in the penultimate nucleotide at the $3^{\prime}$ end of their target-specific sequences (GG, AG, CG, or UG). We found that Cas9 editing is more robust using RNPs than using DNA expression vectors. GG guides are crucial for successful editing using DNA expression vectors and are the most effective guides when using RNPs.

Fifth, we expanded the repertoire of easily scorable coconversion markers used to identify Cas9-edited animals that likely also carry edits in targets of interest. The markers dramatically narrow the search for edited loci that fail to cause visible phenotypes. The $C$. elegans marker zen-4 is selectable, causing edited animals to thrive in a population of temperature-sensitive lethal mutants. The second marker ben-1 serves as a co-conversion marker appropriate for diverse nematode species. Cas9-induced ben-1 mutations confer dominant resistance to the drug benomyl in both $C$. elegans and $C$. briggsae, allowing animals of both species to be mobile rather than paralyzed in the presence of the drug.

Sixth, we explored the timing, location, frequency, sex dependence, and categories of DSB repair events. For this purpose, we designed allele-specific targets of Cas9 to be contributed during mating from either male or hermaphrodite germ cells. We found that male sperm DNA is generally more permissive to Cas9 editing than DNA from hermaphrodite germ cells. Furthermore, the frequency of recovering repair events in the target gene of interest is higher if editable alleles of both the target gene and co-conversion marker are introduced from the same parent during mating. Lastly, both imprecise repair and HDR from either exogenous repair templates or homologous chromosomes occur at unexpectedly high frequencies after fertilization in embryos.

\section{Acknowledgments}

We thank T. Cline, A. Villeneuve, and members of the Meyer laboratory for insightful discussions and D. Stalford for assistance with figures. Some strains in this study were provided by the Caenorhabditis Genetics Center, which is funded by the National Institutes of Health (NIH) Office of Research Infrastructure Programs (P40 OD-010440). A.F.S. was supported by NIH grant R15 GM-117548 and the Center for Gene Regulation in Health and Disease. B.J.M. was supported in part by NIH grant R01 GM-030702. B.J.M. is an investigator of the Howard Hughes Medical Institute.

\section{Literature Cited}

Anders, C., O. Niewoehner, A. Duerst, and M. Jinek, 2014 Structural basis of PAM-dependent target DNA recognition by the Cas9 endonuclease. Nature 513: 569-573. https://doi.org/10. 1038/nature13579

Arribere, J. A., R. T. Bell, B. X. Fu, K. L. Artiles, P. S. Hartman et al., 2014 Efficient marker-free recovery of custom genetic modifications with CRISPR/Cas9 in Caenorhabditis elegans. Genetics 198: 837-846. https://doi.org/10.1534/genetics.114.169730

Blasco, R. B., E. Karaca, C. Ambrogio, T. C. Cheong, E. Karayol et al., 2014 Simple and rapid in vivo generation of chromosomal rearrangements using CRISPR/Cas9 technology. Cell Rep. 9: 1219-1227. https://doi.org/10.1016/j.celrep.2014.10.051

Bothmer, A., T. Phadke, L. A. Barrera, C. M. Margulies, C. S. Lee et al., 2017 Characterization of the interplay between DNA repair and CRISPR/Cas9-induced DNA lesions at an endogenous locus. Nat. Commun. 8: 13905. https://doi.org/10.1038/ncomms13905

Brenner, S., 1974 The genetics of Caenorhabditis elegans. Genetics 77: 71-94.

Carroll, D., 2014 Genome engineering with targetable nucleases. Annu. Rev. Biochem. 83: 409-439. https://doi.org/10.1146/annurev-biochem-060713-035418

Chalfie, M., and J. N. Thomson, 1982 Structural and functional diversity in the neuronal microtubules of Caenorhabditis elegans. J. Cell Biol. 93: 15-23. https://doi.org/10.1083/jcb.93.1.15

Chandrasegaran, S., and D. Carroll, 2016 Origins of programmable nucleases for genome engineering. J. Mol. Biol. 428: 963989. https://doi.org/10.1016/j.jmb.2015.10.014

Chen, X., F. Xu, C. Zhu, J. Ji, X. Zhou et al., 2014 Dual sgRNAdirected gene knockout using CRISPR/Cas9 technology in Caenorhabditis elegans. Sci. Rep. 4: 7581. https://doi.org/10. 1038/srep07581

Cho, S. W., J. Lee, D. Carroll, J. S. Kim, and J. Lee, 2013 Heritable gene knockout in Caenorhabditis elegans by direct injection of Cas9-sgRNA ribonucleoproteins. Genetics 195: 1177-1180. https://doi.org/10.1534/genetics.113.155853

Choi, P. S., and M. Meyerson, 2014 Targeted genomic rearrangements using CRISPR/Cas technology. Nat. Commun. 5: 3728. https://doi.org/10.1038/ncomms4728

Davis, L., and N. Maizels, 2014 Homology-directed repair of DNA nicks via pathways distinct from canonical double-strand break repair. Proc. Natl. Acad. Sci. USA 111: E924-E932. https://doi. org/10.1073/pnas.1400236111

Davis, L., and N. Maizels, 2016 Two distinct pathways support gene correction by single-stranded donors at DNA nicks. Cell Rep. 17: 1872-1881. https://doi.org/10.1016/j.celrep.2016.10.049

Dejima, K., S. Hori, S. Iwata, Y. Suehiro, S. Yoshina et al., 2018 An aneuploidy-free and structurally defined balancer chromosome toolkit for Caenorhabditis elegans. Cell Rep. 22: 232-241. https://doi.org/10.1016/j.celrep.2017.12.024

Dickinson, D. J., J. D. Ward, D. J. Reiner, and B. Goldstein, 2013 Engineering the Caenorhabditis elegans genome using Cas9-triggered homologous recombination. Nat. Methods 10: 1028-1034. https://doi.org/10.1038/nmeth.2641

Dokshin, G. A., K. S. Ghanta, K. M. Piscopo, and C. C. Mello, 2018 Robust genome editing with short single-stranded and long, partially single-stranded DNA donors in Caenorhabditis elegans. Genetics 210: 781-787. https://doi.org/10.1534/genetics.118.301532

Dowdy, S. F., 2017 Overcoming cellular barriers for RNA therapeutics. Nat. Biotechnol. 35: 222-229. https://doi.org/10.1038/ nbt.3802

Driscoll, M., E. Dean, E. Reilly, E. Bergholz, and M. Chalfie, 1989 Genetic and molecular analysis of a Caenorhabditis elegans beta-tubulin that conveys benzimidazole sensitivity. J. Cell Biol. 109: 2993-3003. https://doi.org/10.1083/jcb.109.6.2993 
Farboud, B., and B. J. Meyer, 2015 Dramatic enhancement of genome editing by CRISPR/Cas9 through improved guide RNA design. Genetics 199: 959-971. https://doi.org/10.1534/ genetics.115.175166

Filler Hayut, S., C. Melamed Bessudo, and A. A. Levy, 2017 Targeted recombination between homologous chromosomes for precise breeding in tomato. Nat. Commun. 8: 15605. https://doi.org/10.1038/ncomms15605

Friedland, A. E., Y. B. Tzur, K. M. Esvelt, M. P. Colaiacovo, G. M. Church et al., 2013 Heritable genome editing in C. elegans via a CRISPR-Cas9 system. Nat. Methods 10: 741-743. https://doi. org/10.1038/nmeth.2532

Garneau, J. E., M. E. Dupuis, M. Villion, D. A. Romero, R. Barrangou et al., 2010 The CRISPR/Cas bacterial immune system cleaves bacteriophage and plasmid DNA. Nature 468: 67-71. https://doi. org/10.1038/nature09523

Ghezraoui, H., M. Piganeau, B. Renouf, J. B. Renaud, A. Sallmyr et al., 2014 Chromosomal translocations in human cells are generated by canonical nonhomologous end-joining. Mol. Cell 55: 829-842. https://doi.org/10.1016/j.molcel.2014.08.002

Gibson, D. G., 2011 Enzymatic assembly of overlapping DNA fragments. Methods Enzymol. 498: 349-361. https://doi.org/ 10.1016/B978-0-12-385120-8.00015-2

Hendel, A., R. O. Bak, J. T. Clark, A. B. Kennedy, D. E. Ryan et al., 2015 Chemically modified guide RNAs enhance CRISPR-Cas genome editing in human primary cells. Nat. Biotechnol. 33: 985-989. https://doi.org/10.1038/nbt.3290

Iwata, S., S. Yoshina, Y. Suehiro, S. Hori, and S. Mitani, 2016 Engineering new balancer chromosomes in C. elegans via CRISPR/Cas9. Sci. Rep. 6: 33840. https://doi.org/10.1038/ srep33840

Jantsch-Plunger, V., P. Gonczy, A. Romano, H. Schnabel, D. Hamill et al., 2000 CYK-4: a Rho family gtpase activating protein (GAP) required for central spindle formation and cytokinesis. J. Cell Biol. 149: 1391-1404. https://doi.org/10.1083/jcb.149.7.1391

Jasin, M., and J. E. Haber, 2016 The democratization of gene editing: insights from site-specific cleavage and double-strand break repair. DNA Repair (Amst.) 44: 6-16. https://doi.org/ 10.1016/j.dnarep.2016.05.001

Jiang, F., and J. A. Doudna, 2017 CRISPR-Cas9 structures and mechanisms. Annu. Rev. Biophys. 46: 505-529. https://doi. org/10.1146/annurev-biophys-062215-010822

Jiang, W., X. Zhao, T. Gabrieli, C. Lou, Y. Ebenstein et al., 2015 Cas9-assisted targeting of CHromosome segments CATCH enables one-step targeted cloning of large gene clusters. Nat. Commun. 6: 8101. https://doi.org/10.1038/ncomms9101

Jinek, M., K. Chylinski, I. Fonfara, M. Hauer, J. A. Doudna et al., 2012 A programmable dual-RNA-guided DNA endonuclease in adaptive bacterial immunity. Science 337: 816-821. https://doi. org/10.1126/science.1225829

Joung, J. K., and J. D. Sander, 2013 TALENs: a widely applicable technology for targeted genome editing. Nat. Rev. Mol. Cell Biol. 14: 49-55. https://doi.org/10.1038/nrm3486

Kan, Y., B. Ruis, S. Lin, and E. A. Hendrickson, 2014 The mechanism of gene targeting in human somatic cells. PLoS Genet. 10: e1004251. https://doi.org/10.1371/journal.pgen.1004251

Kan, Y., B. Ruis, T. Takasugi, and E. A. Hendrickson, 2017 Mechanisms of precise genome editing using oligonucleotide donors. Genome Res. 27: 1099-1111. https://doi.org/10.1101/gr.214775. 116

Katic, I., and H. Grosshans, 2013 Targeted heritable mutation and gene conversion by Cas9-CRISPR in Caenorhabditis elegans. Genetics 195: 1173-1176. https://doi.org/10.1534/genetics.113.155754

Katic, I., L. Xu, and R. Ciosk, 2015 CRISPR/Cas9 genome editing in Caenorhabditis elegans: evaluation of templates for homologymediated repair and knock-ins by homology-independent DNA repair. G3 (Bethesda) 5: 1649-1656. https://doi.org/10.1534/ g3.115.019273

Kim, H., T. Ishidate, K. S. Ghanta, M. Seth, D. Conte, Jr. et al., 2014 A co-CRISPR strategy for efficient genome editing in Caenorhabditis elegans. Genetics 197: 1069-1080. https://doi. org/10.1534/genetics.114.166389

Knott, G. J., and J. A. Doudna, 2018 CRISPR-Cas guides the future of genetic engineering. Science 361: 866-869. https://doi. org/10.1126/science.aat5011

Levy, A. D., J. Yang, and J. M. Kramer, 1993 Molecular and genetic analyses of the Caenorhabditis elegans dpy-2 and dpy-10 collagen genes: a variety of molecular alterations affect organismal morphology. Mol. Biol. Cell 4: 803-817. https://doi.org/ $10.1091 / \mathrm{mbc} .4 .8 .803$

Lo, T. W., C. S. Pickle, S. Lin, E. J. Ralston, M. Gurling et al., 2013 Precise and heritable genome editing in evolutionarily diverse nematodes using TALENs and CRISPR/Cas9 to engineer insertions and deletions. Genetics 195: 331-348. https://doi. org/10.1534/genetics.113.155382

Ma, H., N. Marti-Gutierrez, S. W. Park, J. Wu, Y. Lee et al., 2017 Correction of a pathogenic gene mutation in human embryos. Nature 548: 413-419. https://doi.org/10.1038/nature23305

Maddalo, D., E. Manchado, C. P. Concepcion, C. Bonetti, J. A. Vidigal et al., 2014 In vivo engineering of oncogenic chromosomal rearrangements with the CRISPR/Cas9 system. Nature 516: 423-427 [corrigenda: Nature 524: 502 (2015)]. https:// doi.org/10.1038/nature13902

Mali, P., K. M. Esvelt, and G. M. Church, 2013a Cas9 as a versatile tool for engineering biology. Nat. Methods 10: 957-963. https://doi.org/10.1038/nmeth.2649

Mali, P., L. Yang, K. M. Esvelt, J. Aach, M. Guell et al., 2013b RNA-guided human genome engineering via Cas9. Science 339: 823-826. https://doi.org/10.1126/science.1232033

Mojica, F. J., C. Díez-Villaseñor, J. García-Martínez, and C. Almendros, 2009 Short motif sequences determine the targets of the prokaryotic CRISPR defence system. Microbiology 155: 733-740. https:// doi.org/10.1099/mic.0.023960-0

Paix, A., Y. Wang, H. E. Smith, C. Y. Lee, D. Calidas et al., 2014 Scalable and versatile genome editing using linear DNAs with microhomology to Cas9 Sites in Caenorhabditis elegans. Genetics 198: 1347-1356. https://doi.org/10.1534/genetics.114.170423

Paix, A., A. Folkmann, D. Rasoloson, and G. Seydoux, 2015 High efficiency, homology-directed genome editing in Caenorhabditis elegans using CRISPR-Cas9 ribonucleoprotein complexes. Genetics 201: 47-54. https://doi.org/10.1534/genetics.115.179382

Paix, A., A. Folkmann, D. H. Goldman, H. Kulaga, M. J. Grzelak et al., 2017 Precision genome editing using synthesis-dependent repair of Cas9-induced DNA breaks. Proc. Natl. Acad. Sci. USA 114: E10745-E10754. https://doi.org/10.1073/pnas.1711979114

Powers, J., O. Bossinger, D. Rose, S. Strome, and W. Saxton, 1998 A nematode kinesin required for cleavage furrow advancement. Curr. Biol. 8: 1133-1136. https://doi.org/10.1016/ S0960-9822(98)70470-1

Raich, W. B., A. N. Moran, J. H. Rothman, and J. Hardin, 1998 Cytokinesis and midzone microtubule organization in Caenorhabditis elegans require the kinesin-like protein ZEN-4. Mol. Biol. Cell 9: 2037-2049. https://doi.org/10.1091/mbc.9. 8.2037

Richardson, C. D., K. R. Kazane, S. J. Feng, E. Zelin, N. L. Bray et al., 2018 CRISPR-Cas9 genome editing in human cells occurs via the Fanconi anemia pathway. Nat. Genet. 50: 1132-1139. https://doi.org/10.1038/s41588-018-0174-0

Sekelsky, J., 2017 DNA repair in Drosophila: mutagens, models, and missing genes. Genetics 205: 471-490. https://doi.org/ 10.1534/genetics.116.186759 
Severson, A. F., and B. Bowerman, 2002 Cytokinesis: closing in on the central spindle. Dev. Cell 2: 4-6. https://doi.org/10.1016/ S1534-5807(01)00113-7

Severson, A. F., D. R. Hamill, J. C. Carter, J. Schumacher, and B. Bowerman, 2000 The aurora-related kinase AIR-2 recruits ZEN-4/CeMKLP1 to the mitotic spindle at metaphase and is required for cytokinesis. Curr. Biol. 10: 1162-1171. https://doi. org/10.1016/S0960-9822(00)00715-6

Stephenson, A. A., A. T. Raper, and Z. Suo, 2018 Bidirectional degradation of DNA cleavage products catalyzed by CRISPR/ Cas9. J. Am. Chem. Soc. 140: 3743-3750. https://doi.org/10. 1021/jacs.7b13050

Sternberg, S. H., S. Redding, M. Jinek, E. C. Greene, and J. A. Doudna, 2014 DNA interrogation by the CRISPR RNA-guided endonuclease Cas9. Nature 507: 62-67. https://doi.org/10.1038/ nature13011

Sternberg, S. H., B. LaFrance, M. Kaplan, and J. A. Doudna, 2015 Conformational control of DNA target cleavage by CRISPR-Cas9. Nature 527: 110-113. https://doi.org/10.1038/ nature 15544

Suzuki, T., M. Asami, and A. C. Perry, 2014 Asymmetric parental genome engineering by Cas 9 during mouse meiotic exit. Sci. Rep. 4: 7621. https://doi.org/10.1038/srep07621

Tzur, Y. B., A. E. Friedland, S. Nadarajan, G. M. Church, J. A. Calarco et al., 2013 Heritable custom genomic modifications in Caenorhabditis elegans via a CRISPR-Cas9 system. Genetics 195: 1181-1185. https://doi.org/10.1534/genetics.113.156075

Urnov, F. D., E. J. Rebar, M. C. Holmes, H. S. Zhang, and P. D. Gregory, 2010 Genome editing with engineered zinc finger nucleases. Nat. Rev. Genet. 11: 636-646. https://doi.org/10.1038/nrg2842

van Overbeek, M., D. Capurso, M. M. Carter, M. S. Thompson, E. Frias et al., 2016 DNA repair profiling reveals nonrandom outcomes at
Cas9-mediated breaks. Mol. Cell 63: 633-646. https://doi.org/ 10.1016/j.molcel.2016.06.037

Waaijers, S., V. Portegijs, J. Kerver, B. B. Lemmens, M. Tijsterman et al., 2013 CRISPR/Cas9-targeted mutagenesis in Caenorhabditis elegans. Genetics 195: 1187-1191. https://doi.org/10.1534/ genetics.113.156299

Ward, J. D., 2015 Rapid and precise engineering of the Caenorhabditis elegans genome with lethal mutation coconversion and inactivation of NHEJ repair. Genetics 199: 363-377. https://doi.org/10.1534/genetics.114.172361

Witte, H., E. Moreno, C. Rödelsperger, J. Kim, J. S. Kim et al., 2015 Gene inactivation using the CRISPR/Cas9 system in the nematode Pristionchus pacificus. Dev. Genes Evol. 225: 5562. https://doi.org/10.1007/s00427-014-0486-8

Wood, A. J., T. W. Lo, B. Zeitler, C. S. Pickle, E. J. Ralston et al., 2011 Targeted genome editing across species using ZFNs and TALENs. Science 333: 307. https://doi.org/10.1126/science. 1207773

Wu, Y., D. Liang, Y. Wang, M. Bai, W. Tang et al., 2013 Correction of a genetic disease in mouse via use of CRISPR-Cas9. Cell Stem Cell 13: 659-662. https://doi.org/10.1016/j.stem.2013. 10.016

Zhao, P., Z. Zhang, H. Ke, Y. Yue, and D. Xue, 2014 Oligonucleotide-based targeted gene editing in C. elegans via the CRISPR/Cas9 system. Cell Res. 24: 247-250. https://doi.org/10.1038/cr.2014.9

Zuo, Z., and J. Liu, 2016 Cas9-catalyzed DNA cleavage generates staggered ends: evidence from molecular dynamics simulations. Sci. Rep. 5: 37584. https://doi.org/10.1038/srep37584

Communicating editor: D. Greenstein 